TRANSACTIONS OF THE AMERICAN MATHEMATICAL SOCIETY

Volume 348, Number 10, October 1996

\title{
SEPARABLE BANACH SPACE THEORY NEEDS STRONG SET EXISTENCE AXIOMS
}

\author{
A. JAMES HUMPHREYS AND STEPHEN G. SIMPSON
}

\begin{abstract}
We investigate the strength of set existence axioms needed for separable Banach space theory. We show that a very strong axiom, $\Pi_{1}^{1}$ comprehension, is needed to prove such basic facts as the existence of the weak-* closure of any norm-closed subspace of $\ell_{1}=c_{0}^{*}$. This is in contrast to earlier work $[6,4,7,23,22]$ in which theorems of separable Banach space theory were proved in very weak subsystems of second order arithmetic, subsystems which are conservative over Primitive Recursive Arithmetic for $\Pi_{2}^{0}$ sentences. En route to our main results, we prove the Krein-Šmulian theorem in $\mathrm{ACA}_{0}$, and we give a new, elementary proof of a result of McGehee on weak-* sequential closure ordinals.
\end{abstract}

\section{INTRODUCTION}

This paper is part of an ongoing study of the role of set existence axioms in the foundations of mathematics. The ongoing study has been carried out in the context of subsystems of second order arithmetic, under the slogan Reverse Mathematics $[12,3,23]$. We continue this program here by examining the role of strong set existence axioms in separable Banach space theory. We show that a very strong set existence axiom is needed in order to prove basic results concerning the weak-* topology on the dual of a separable Banach space.

The results in this paper are related to earlier work of Brown and Simpson $[6,4,7,23]$ and Shioji and Tanaka [22]. The earlier work shows that the basic notions of separable Banach space theory can be developed in very weak subsystems of second order arithmetic, and that many basic results can be proved in such systems. Specifically, the Hahn-Banach theorem and a version of the Schauder fixed point theorem are provable in $\mathrm{WKL}_{0}$; the Banach-Steinhaus theorem is provable in $\mathrm{RCA}_{0}$; and versions of the Open Mapping and Closed Graph theorems are provable in $\mathrm{RCA}_{0}^{+}$. The set existence axioms of these three subsystems of second order arithmetic are very weak, in the sense that the systems themselves are conservative over Primitive Recursive Arithmetic for $\Pi_{2}^{0}$ sentences (see Chapter IX of [23]). In particular, the mentioned systems are considerably weaker than first order arithmetic. Thus the results of Brown and Simpson [6, 4, 7] may have tended to support the

Received by the editors July 10, 1995.

1991 Mathematics Subject Classification. Primary 03F35; Secondary 46B10, 46 B45.

Key words and phrases. Reverse mathematics, separable Banach space theory, weak-* topology, closure ordinals, Krein-Šmulian theorem.

This research was partially supported by NSF grant DMS-9303478. We would also like to thank our colleague Robert E. Huff for showing us his unpublished notes on the Krein-Šmulian theorem, and the referee for helpful comments which improved the exposition of this paper. 
opinion that only very weak set existence axioms are needed for separable Banach space theory. Our main results here, Theorems 5.6 and 5.7 below, provide a counterexample to that opinion and a departure from Brown-Simpson-Shioji-Tanaka. Namely, Theorems 5.6 and 5.7 show that a very strong set existence axiom, $\Pi_{1}^{1}$ comprehension, is needed in order to prove basic facts such as the existence of the weak-* closure of any norm-closed subspace of $\ell_{1}=c_{0}^{*}$. Thus $\Pi_{1}^{1}$ comprehension is in a sense indispensable for separable Banach space theory. This is significant because $\Pi_{1}^{1}$ comprehension is, of course, much stronger than first order arithmetic.

As a byproduct, we show that the Krein-Šmulian theorem for the dual of a separable Banach space (Theorem 2.7 below) is provable in ACA $_{0}$ (Theorem 4.14 below). We conjecture that the Krein-Šmulian theorem for the dual of a separable Banach space is actually provable in the weaker system $\mathrm{WKL}_{0}$.

Some of our results here may be of interest to readers who are familiar with Banach spaces but do not share our concern with Reverse Mathematics and other foundational issues. Namely, the following Banach space phenomenon may be of independent interest. Let $Z$ be a subspace of the dual of a separable Banach space. Banach and Mazurkiewicz observed that, although the weak-* closure of $Z$ is the same as the weak-* sequential closure of $Z$, it is not necessarily the case that every point of the weak-* closure of $Z$ is the weak-* limit of a sequence of points of $Z$. Indeed, the process of taking weak-* limits of sequences may need to be iterated transfinitely many times in order to obtain the weak-* closure. In a self-contained part of this paper, we obtain a sharp result along these lines. Namely, for each countable ordinal $\alpha$, we obtain an explicit example of a norm-closed, weak-* dense subspace of $\ell_{1}=c_{0}^{*}$ whose weak-* sequential closure ordinal is exactly $\alpha+1$. This result is originally due to McGehee [18], but our examples are different and more elementary.

On the other hand, it is perhaps worth noting that our original motivation for the work here had nothing to do with Banach space theory. Rather, our starting point was another aspect of Reverse Mathematics, specifically the search for necessary uses of strong set existence axioms in classical ("hard") analysis. We began with the thought that, in searching for necessary uses of strong set existence axioms, it would be natural to consider how Cantor was led to the invention or discovery of set theory in the first place. We were struck by the well known historical fact $[9,10]$ that Cantor introduced ordinal numbers in tandem with his study of trigonometric series and the structure of sets of uniqueness; see also Jourdain's essay [8]. Indeed, Cantor's proof that every countable closed set is a set of uniqueness uses transfinite induction on the Cantor-Bendixson rank of such sets. Therefore, from our Reverse Mathematics viewpoint, it is very natural to reexamine these results of Cantor. Although we postpone such reexamination to a future paper, we want to point out that our work here was inspired by a discussion of Kechris and Louveau [14, 13] culminating in a result attributed to Solovay: the Piatetski-Shapiro rank is a $\Pi_{1}^{1}$ rank on the set of closed sets of uniqueness. Since the Piatetski-Shapiro rank is the weak-* sequential closure ordinal of a certain weak-* dense subspace of $\ell_{1}$, our foundational motivation for studying such ordinals is apparent.

We end this introductory section with a brief outline of the rest of the paper. Section 2 reviews the concepts and results of Banach space theory that are important for us here. In particular we review the weak-* topology and define the notion of the weak-* sequential closure ordinal of an arbitrary set in the dual of a separable Banach space. In Section 3 we exhibit the previously mentioned examples 
concerning weak-* sequential closure ordinals, using the concept of a smooth tree. These two sections, Sections 2 and 3, are intended to form a self-contained unit which should be accessible to anyone who is familiar with the notion of a Banach space. Our discussion of subsystems of second order arithmetic does not get under way until Section 4 . We begin that section by reviewing the definitions and results from Brown-Simpson $[6,4,7,23]$ that we shall need. We then discuss the weak-* topology and related notions in the Brown-Simpson context. We end Section 4 by proving our version of the Krein-Šmulian theorem within $\mathrm{ACA}_{0}$. Finally, in Section 5, we state and prove our main theorem, concerning the need for $\Pi_{1}^{1}$ comprehension. The ideas of Section 3 are used in the proof of the main theorem in Section 5.

\section{BANACH SPACE PRELIMINARIES}

The purpose of this section is to review some well-known concepts and results from separable Banach space theory. Our focus is the weak-* topology on the dual of a separable Banach space. A reference for most of this material is Chapter V of Dunford and Schwartz [11].

Let $X$ be a Banach space. The weak topology on $X$ is the weakest topology such that every bounded linear functional on $X$ is continuous. The dual space of $X$ is the space $X^{*}$ of all bounded linear functionals on $X$. The norm of $x^{*} \in X^{*}$ is defined by

$$
\left\|x^{*}\right\|=\sup \left\{\left|x^{*}(x)\right| \mid\|x\| \leq 1\right\} .
$$

The weak-* topology on $X^{*}$ is the weakest topology such that for all $x \in X$ the functional $x^{*} \mapsto x^{*}(x)$ is continuous. The weak-* closure of a set $Z \subseteq X^{*}$ is denoted $c l *(Z)$. Note that about any point $x_{0}^{*} \in X^{*}$ there is a weak-* neighborhood basis consisting of all sets of the form

$$
\left\{x^{*} \in X^{*}|| x^{*}\left(x_{1}\right)-x_{0}^{*}\left(x_{1}\right)|<1, \ldots,| x^{*}\left(x_{n}\right)-x_{0}^{*}\left(x_{n}\right) \mid<1\right\}
$$

for some finite set $x_{1}, \ldots, x_{n} \in X$.

A key theorem concerning the weak-* topology is:

Theorem 2.1 (Banach-Alaoglu). For any $r>0$, the closed ball

$$
B_{r}\left(X^{*}\right)=\left\{x^{*} \in X^{*} \mid\left\|x^{*}\right\| \leq r\right\}
$$

is weak-* closed and weak-* compact. Furthermore, if $X$ is separable then $B_{r}\left(X^{*}\right)$ is weak-* metrizable.

Proof. See [11], page 424.

In considering weak-* sequential convergence, an important fact to keep in mind is the following consequence of the Banach-Steinhaus theorem:

Theorem 2.2. Let $\left\{x_{n}^{*} \mid n \in \mathbb{N}\right\}$ be a countable set of points in $X^{*}$. If $\sup _{n}\left|x_{n}^{*}(x)\right|$ $<\infty$ for all $x \in X$, then $\sup _{n}\left\|x_{n}^{*}\right\|<\infty$.

Proof. See [11], page 66.

Corollary 2.3. If $x_{n}^{*} \rightarrow x^{*}$ in the weak-* topology in $X^{*}$, then $\left\{x_{n}^{*} \mid n \in \mathbb{N}\right\}$ is bounded, i.e., $\left\{x_{n}^{*} \mid n \in \mathbb{N}\right\} \subseteq B_{r}\left(X^{*}\right)$ for some $0<r<\infty$.

A set $Z \subseteq X^{*}$ is said to be weak-* sequentially closed if it is closed under weak-* limits of sequences, i.e., $x_{n}^{*} \rightarrow x^{*}$ weak- $*$ and $x_{n}^{*} \in Z$ for all $n \in \mathbb{N}$ imply $x^{*} \in Z$. 
Corollary 2.4. A set $Z \subseteq X^{*}$ is weak-* sequentially closed if and only if $Z \cap$ $B_{r}\left(X^{*}\right)$ is weak-* sequentially closed for all $r>0$.

For an arbitrary set $Z \subseteq X^{*}$, being weak-* sequentially closed is not in general equivalent to being weak-* closed. In particular, the weak-* topology is not in general metrizable, even when $X$ is separable. This is shown by the following theorem.

Theorem 2.5. Let $X$ be an infinite-dimensional separable Banach space. Then we can find a countable set $Z \subset X^{*}$ such that $Z$ is weak-* sequentially closed yet weak-* dense in $X^{*}$.

Proof. We first prove the following lemma.

Lemma 2.6. Let $X$ be an infinite-dimensional Banach space. Then we can find a sequence of points $\left\langle x_{k} \mid k \in \mathbb{N}\right\rangle$ in $X$ such that $\lim _{k}\left\|x_{k}\right\|=\infty$ and $\left\|x_{k}\right\|>1$ for all $k \in \mathbb{N}$, yet 0 belongs to the weak closure of $\left\{x_{k} \mid k \in \mathbb{N}\right\}$.

Proof. For any finite set $F \subset X^{*},\left\{x \in X \mid y^{*}(x)=0\right.$ for all $\left.y^{*} \in F\right\}$ is a subspace of $X$ of codimension at most the cardinality of $F$, and hence in particular it intersects $\{x \in X \mid\|x\|>n\}$ for each $n \in \mathbb{N}$. By the Banach-Alaoglu theorem, $B_{n}\left(X^{*}\right)$ is weak-* compact, and so $B_{n}\left(X^{*}\right)^{n}$ is weak-* compact as well. Thus for each $n$ we can find a finite set $G_{n} \subset\{x \in X \mid\|x\|>n\}$ such that, for each finite set $F \subset B_{n}\left(X^{*}\right)$ of cardinality $n,\left\{x \in X|| y^{*}(x) \mid<1\right.$ for all $\left.y^{*} \in F\right\}$ intersects $G_{n}$. Letting $\left\{x_{k} \mid k \in \mathbb{N}\right\}$ be an enumeration without repetition of $\bigcup_{n=1}^{\infty} G_{n}$, we obtain the desired sequence.

Proof of Theorem 2.5. Since $X$ is separable, it follows by Theorem 2.1 that $X^{*}$ is weak-* separable, so let $\left\{x_{n}^{*} \mid n \in \mathbb{N}\right\}$ be a countable weak-* dense subset of $X^{*}$. By the preceding lemma, for each $n$ we can find a sequence $\left\langle z_{n k}^{*} \mid k \in \mathbb{N}\right\rangle$ such that $\left\|z_{n k}^{*}\right\|>n$ for all $k$ and $\lim _{k}\left\|z_{n k}^{*}\right\|=\infty$ and $x_{n}^{*}$ belongs to the weak, and hence weak-*, closure of $\left\{z_{n k}^{*} \mid k \in \mathbb{N}\right\}$. Thus $Z=\left\{z_{n k}^{*} \mid n, k \in \mathbb{N}\right\}$ is a countable set which is weak-* dense in $X^{*}$. On the other hand, for each $n, Z \cap B_{n}\left(X^{*}\right)$ is finite, so by Corollary $2.4 Z$ is weak-* sequentially closed.

The previous theorem shows that a set in $X^{*}$ can be weak-* sequentially closed yet far from weak-* closed, even when $X$ is separable. Nevertheless, it turns out that for convex sets in $X^{*}$, being weak-* sequentially closed is equivalent to being weak-* closed, provided $X$ is separable. We shall obtain this result as a consequence of the following well-known theorem:

Theorem 2.7 (Krein-Šmulian). Let $X$ be a Banach space. A convex set in $X^{*}$ is weak-* closed if and only if its intersection with $B_{r}\left(X^{*}\right)$ is weak-* closed for every $r>0$.

Proof. See [11], page 429.

The special case of the Krein-Šmulian theorem for subspaces of $X^{*}$ is originally due to Banach ([2], page 124):

Corollary 2.8 (Banach). A subspace of $X^{*}$ is weak-* closed if and only if its intersection with $B_{1}\left(X^{*}\right)$ is weak-* closed.

Proof. For a subspace $Z$ of $X^{*}$, we have $Z \cap B_{r}\left(X^{*}\right)=r\left(Z \cap B_{1}\left(X^{*}\right)\right)$. Hence $Z \cap B_{r}\left(X^{*}\right)$ is weak-* closed for all $r$ if and only if $Z \cap B_{1}\left(X^{*}\right)$ is weak-* closed. The desired result follows immediately from the Krein-Šmulian theorem. 
A set $Z$ in $X^{*}$ is said to be bounded-weak-* closed if $Z \cap B_{r}\left(X^{*}\right)$ is weak-*closed for all $r>0$. This defines yet another topology on $X^{*}$, the bounded-weak-* topology. By the Banach-Alaoglu theorem, weak-* closed sets are bounded-weak-* closed, but the converse does not hold in general. We can paraphrase the KreinŠmulian theorem by saying that a convex set in $X^{*}$ is weak-* closed if and only if it is bounded-weak-* closed.

Lemma 2.9. Let $X$ be a separable Banach space. A set $Z \subseteq X^{*}$ is bounded-weak-* closed if and only if it is weak-* sequentially closed.

Proof. By definition, $Z$ is bounded-weak-* closed if and only if $Z \cap B_{r}\left(X^{*}\right)$ is weak-* closed for all $r$. Since $X$ is separable, we have by Theorem 2.1 that $B_{r}\left(X^{*}\right)$ is weak-* compact and weak-* metrizable. Hence, for all $r, Z \cap B_{r}\left(X^{*}\right)$ is weak-* closed if and only if $Z \cap B_{r}\left(X^{*}\right)$ is weak-* sequentially closed. By Corollary 2.4 it now follows that $Z \cap B_{r}\left(X^{*}\right)$ is weak-* closed for all $r$ if and only if $Z$ is weak-* sequentially closed. This completes the proof.

We now obtain the desired result:

Theorem 2.10. Let $X$ be a separable Banach space. A convex set $Z \subseteq X^{*}$ is weak-* closed if and only if it is weak-* sequentially closed.

Proof. Immediate from Theorem 2.7 plus Lemma 2.9.

Again, the special case when $Z$ is a subspace of $X^{*}$ is due to Banach ([2], page 124):

Corollary 2.11 (Banach). Let $X$ be a separable Banach space. A subspace $Z$ of $X^{*}$ is weak-* closed if and only if it is weak-* sequentially closed.

We now turn to a discussion of weak-* sequential closure ordinals. For an arbitrary set $Z \subseteq X^{*}$, let $Z^{\prime}$ denote the set of weak-* limits of sequences from $Z$. Define a transfinite sequence of sets $Z^{(\alpha)}, \alpha$ an ordinal, by

$$
\begin{aligned}
Z^{(0)} & =Z, \\
Z^{(\alpha+1)} & =\left(Z^{(\alpha)}\right)^{\prime}, \\
Z^{(\delta)} & =\bigcup_{\alpha<\delta} Z^{(\alpha)} \quad \text { for } \delta \text { a limit ordinal. }
\end{aligned}
$$

Clearly $\alpha<\beta$ implies $Z^{(\alpha)} \subseteq Z^{(\beta)}$. Define ord $(Z)$ to be the least ordinal $\alpha$ such that $Z^{(\alpha+1)}=Z^{(\alpha)}$. Thus $Z^{(\operatorname{ord}(Z))}$ is the weak-* sequential closure of $Z$. We call $\operatorname{ord}(Z)$ the closure ordinal of $Z$. Note that if $Z$ is convex, then $Z^{(\alpha)}$ is convex for all $\alpha$; also, by the previous theorem $Z^{(\operatorname{ord}(Z))}=c l *(Z)$, the weak-* closure of $Z$.

In the remainder of this section and the next section, we shall prove some results which completely answer the question of which ordinals can arise as closure ordinals of subspaces of $X^{*}$ when $X$ is a separable Banach space. This question was first answered completely by McGehee [18] and Sarason [19, 20, 21].

Lemma 2.12. If $X$ is a separable Banach space, then for any set $Z \subseteq X^{*}$ the closure ordinal $\operatorname{ord}(Z)$ is countable.

Proof. For $r>0$ and $\alpha$ an ordinal, let $C_{r}^{\alpha}$ be the weak-* closure of $Z^{(\alpha)} \cap B_{r}\left(X^{*}\right)$. For fixed $r>0$, the sets $C_{r}^{\alpha}$ form an increasing, transfinite sequence of compact 
subsets of a compact metric space, namely $B_{r}\left(X^{*}\right)$ with the weak-* topology (Theorem 2.1). This transfinite sequence must therefore stabilize at some countable ordinal. Since $C_{r}^{\alpha} \subseteq Z^{(\alpha+1)} \cap B_{r}\left(X^{*}\right) \subseteq C_{r}^{\alpha+1}$ for all $\alpha$, it follows that the transfinite sequence $Z^{(\alpha)} \cap B_{r}\left(X^{*}\right)$ also stabilizes at a countable ordinal, call it $\alpha_{r}$. Put $\alpha=\sup \left\{\alpha_{n} \mid n \in \mathbb{N}\right\}$. Then $\alpha$ is a countable ordinal. We claim that $Z^{(\alpha)}$ is weak-* sequentially closed. To see this, suppose that $x^{*}$ is the weak-* limit of a sequence $\left\langle x_{k}^{*} \mid k \in \mathbb{N}\right\rangle$ from $Z^{(\alpha)}$. By Corollary 2.3, there exists $n \in \mathbb{N}$ such that $x_{k}^{*} \in B_{n}\left(X^{*}\right)$ for all $k \in \mathbb{N}$. Hence $x^{*} \in Z^{(\alpha+1)} \cap B_{n}\left(X^{*}\right)=Z^{(\alpha)} \cap B_{n}\left(X^{*}\right)$, so in particular $x^{*} \in Z^{(\alpha)}$ and our claim is proved. Thus $\operatorname{ord}(Z) \leq \alpha<\omega_{1}$, i.e., $\operatorname{ord}(Z)$ is countable.

Specializing to subspaces of $X^{*}$, we can say more:

Theorem 2.13. Let $X$ be a separable Banach space and let $Z$ be a subspace of $X^{*}$. Then the closure ordinal $\operatorname{ord}(Z)$ is a countable successor ordinal, unless $Z$ is already weak-* closed, in which case $\operatorname{ord}(Z)=0$.

Proof. By the previous lemma, ord $(Z)$ is countable. Suppose that $c l *(Z)=Z^{(\delta)}=$ $\bigcup_{\alpha<\delta} Z^{(\alpha)}$, for some countable limit ordinal $\delta$. As $c l *(Z)$ is norm closed, this implies that $c l *(Z)=\bigcup_{\alpha<\delta} c l\left(Z^{(\alpha)}\right)$, where $c l(Y)$ denotes the norm closure of $Y \subseteq X^{*}$. Thus $c l *(Z)$ is a closed subset of a complete metric space, written as a countable union of closed sets. By the Baire category theorem, there must be an ordinal $\alpha<\delta$ such that $c l\left(Z^{(\alpha)}\right)$ has non-void interior as a subset of $c l *(Z)$, i.e., $\operatorname{cl}\left(Z^{(\alpha)}\right)$ contains the intersection of an open (in norm) ball with $c l *(Z)$. But $\operatorname{cl}\left(Z^{(\alpha)}\right)$ is a subspace of $c l *(Z)$, so it must be all of $c l *(Z)$. Since $c l\left(Z^{(\alpha)}\right) \subseteq Z^{(\alpha+1)}$, it follows that $Z^{(\alpha+1)}=c l *(Z)$, whence $\operatorname{ord}(Z) \neq \delta$. We have now shown that $\operatorname{ord}(Z)$ is not a limit ordinal. Thus $\operatorname{ord}(Z)$ must be either 0 or a successor ordinal. If $\operatorname{ord}(Z)=0$ then $Z$ is weak-* sequentially closed, and hence is weak-* closed by Corollary 2.11. This completes the proof. See also Kechris and Louveau [14], page 157.

It is known that the converse of the previous theorem also holds: For every countable successor ordinal $\alpha+1$, we can find a subspace $Z$ of the dual $X^{*}$ of a separable Banach space $X$ such that $\operatorname{ord}(Z)=\alpha+1$. In the next section we present a proof of this result, with $Z \subseteq \ell_{1}=c_{0}^{*}$ and $Z$ weak- $*$ dense in $\ell_{1}$.

The study of closure ordinals of subspaces of $X^{*}$ has an interesting history. Von Neumann ([24], page 380) exhibits a set $S \subset \ell_{2}$ such that 0 is in the weak closure of $S$ yet no sequence from $S$ converges weakly to 0 . The first example of a subspace $Z$ of $X^{*}$ such that $\operatorname{ord}(Z) \geq 2$ is due to Mazurkiewicz [17]. Banach ([2], pages 209-213) proves that for every $n \in \mathbb{N}$ there is a subspace $Z$ of $\ell_{1}=c_{0}^{*}$ such that $\operatorname{ord}(Z) \geq n$ and states the analogous result for all countable ordinals. For the proof Banach refers to a "forthcoming" paper which seems never to have appeared, and this reference is omitted from the English translation [1]. Later, McGehee [18] proves the stronger result that for each countable ordinal $\alpha$ there exists a weak-* dense subspace of $\ell_{1}=c_{0}^{*}$ whose closure ordinal is exactly $\alpha+1$ (but note that McGehee's notation differs from ours). In the next section we reprove this result of McGehee. Sarason $[19,20,21]$ proves a similar result for the spaces $H^{\infty}$ and $\ell_{\infty}$. In view of the theorem above, these results of McGehee and Sarason are in a sense best possible. While McGehee's proof uses sets of synthesis and uniqueness, our proof in the next section is much more elementary. 


\section{TREes AND subspaces OF $\ell_{1}$}

In this section we prove the following result: For each countable ordinal $\alpha$, there exists a weak-* dense subspace $Z$ of $\ell_{1}=c_{0}^{*}$ such that $\operatorname{ord}(Z)=\alpha+1$. Our proof uses some simple concepts and results concerning trees. We give a self-contained treatment of these auxiliary results.

Definition 3.1. Let Seq denote the set of finite sequences of natural numbers, i.e.,

$$
\text { Seq }=\bigcup_{k=0}^{\infty} \mathbb{N}^{k}=\left\{\left\langle n_{0}, \ldots, n_{k-1}\right\rangle \mid k \in \mathbb{N}, n_{i} \in \mathbb{N} \text { for all } i<k\right\} .
$$

For $s=\left\langle n_{0}, \ldots, n_{k-1}\right\rangle \in$ Seq, we write

$$
s=\langle s(0), s(1), \ldots, s(\operatorname{lh}(s)-1)\rangle,
$$

where $\operatorname{lh}(s)=k$ denotes the length of $s$, and $s(i)=n_{i}$ for all $i<\operatorname{lh}(s)$. In particular \langle\rangle is the empty sequence, the unique sequence of length 0 . For $s, t \in$ Seq we denote by $s\urcorner t$ the concatenation of $s$ and $t$, i.e., the sequence of length $\operatorname{lh}(s)+\operatorname{lh}(t)$ given by

$$
s\urcorner t=\langle s(0), \ldots, s(\operatorname{lh}(s)-1), t(0), \ldots, t(\operatorname{lh}(t)-1)\rangle .
$$

For $s, t \in$ Seq, we write $s \subseteq t$ to mean that $s$ is an initial segment of $t$, i.e., $\operatorname{lh}(s) \leq \operatorname{lh}(t)$ and, for all $i<\operatorname{lh}(s), s(i)=t(i)$. Given $s \in$ Seq, if $s \neq\langle\rangle$ let

$$
s^{\prime}=\langle s(0), s(1), \ldots, s(\operatorname{lh}(s)-2)\rangle \text {, }
$$

i.e., $s^{\prime}$ is the initial segment of $s$ of length $\operatorname{lh}(s)-1$. For $s=\langle\rangle$ we put $s^{\prime}=\langle\rangle^{\prime}=\langle\rangle$.

Definition 3.2. We define a tree to be a nonempty set $T \subseteq$ Seq which is closed under taking initial segments, i.e., for all $s, t \in$ Seq, if $t \in T$ and $s \subseteq t$ then $s \in T$. If $T$ is a tree and $s \in T$, we say that $s$ is a node of $T$. If $s$ is a node of $T$ such that $s^{\frown}\langle n\rangle \notin T$ for all $n \in \mathbb{N}$, then $s$ is called an end node; otherwise $s$ is called an interior node of $T$. Given a tree $T$, a function $f: \mathbb{N} \rightarrow \mathbb{N}$ is called a path through $T$ if for all $n \in \mathbb{N}$ we have $f[n] \in T$, where $f[n]=\langle f(0), f(1), \ldots, f(n-1)\rangle$. A tree $T$ is said to be well-founded if it has no path.

Definition 3.3. If $T$ is a tree, let

$$
T^{\prime}=\left\{t^{\prime} \mid t \in T\right\}=\{\text { interior nodes of } T\} \cup\{\langle\rangle\} .
$$

Note that $T^{\prime}$ is a subtree of $T$. We define a transfinite sequence of subtrees $T^{(\alpha)}$ of $T$ by

$$
\begin{aligned}
T^{(0)} & =T, \\
T^{(\alpha+1)} & =\left(T^{(\alpha)}\right)^{\prime}, \\
T^{(\delta)} & =\bigcap_{\alpha<\delta} T^{(\alpha)} \quad \text { for } \delta \text { a limit ordinal. }
\end{aligned}
$$

Note that $T$ is well-founded if and only if $T^{(\alpha)}=\{\langle\rangle\}$ for some countable ordinal $\alpha$. The least such $\alpha$ is called the height of $T$, denoted $h(T)$. Given a well-founded tree $T$, we define a function $h_{T}:$ Seq $\rightarrow \operatorname{Ord} \cup\{-1\}$ (where Ord denotes the set of countable ordinals) by $h_{T}(s)=-1$ for $s \notin T$ and, for $s \in T, h_{T}(s)=$ the least $\alpha$ such that $s$ is an end node of $T^{(\alpha)}$. In particular $h_{T}(\langle\rangle)=h(T)$. Note that, for all $s \in T, h_{T}(s)=\sup \left\{h_{T}\left(s^{\frown}\langle n\rangle\right)+1 \mid n \in \mathbb{N}\right\}$. 
From the definition above, the height of a well-founded tree is a countable ordinal. The following standard theorem shows that the converse holds as well.

Theorem 3.4. For any countable ordinal $\alpha$, we can construct a well-founded tree $T$ such that $h(T)=\alpha$.

Proof. We prove this by transfinite induction on $\alpha$. For $\alpha=0$ we have $h(\{\langle\rangle\})=0$. For successor ordinals, note that if $h(T)=\alpha$, then $h\left(T^{+}\right)=\alpha+1$ where $T^{+}=\{\langle\rangle\} \cup$ $\left\{\langle 0\rangle^{\wedge} s \mid s \in T\right\}$. Suppose now that $\delta$ is a limit ordinal, say $\delta=\sup \left\{\alpha_{n} \mid n \in \mathbb{N}\right\}$, where $\alpha_{n}<\delta$ for all $n \in \mathbb{N}$. For each $n$ let $T_{n}$ be a tree of height $\alpha_{n}$, and put $T=\{\langle\rangle\} \cup\left\{\langle n\rangle^{\wedge} s \mid n \in \mathbb{N}, s \in T_{n}\right\}$. Then $T$ is well-founded and, for each $n \in \mathbb{N}$, $h_{T}(\langle n\rangle)=\alpha_{n}$. Thus $h(T)=\sup \left\{\alpha_{n}+1 \mid n \in \mathbb{N}\right\}=\delta$.

Definition 3.5. Fix an injection \# : Seq $\rightarrow \mathbb{N}$ with the following properties:

1. $\#(\langle\rangle)=1$;

2. $s \subseteq t$ implies $\#(s) \leq \#(t)$;

3. $m<n$ implies \# $\left(s^{\wedge}\langle m\rangle\right)<\#\left(s^{\curvearrowleft}\langle n\rangle\right)$.

Given $s \in$ Seq we refer to \#(s) as the Gödel number of $s$. To simplify notation in what follows, we shall often identify sequences with their Gödel numbers, i.e., we write $s$ instead of $\#(s)$.

Let $X=c_{0}(\mathrm{Seq})$, the space of sequences of real numbers converging to 0 indexed by Seq. Then we may identify $X^{*}$ with $\ell_{1}($ Seq), the space of absolutely summable sequences of real numbers indexed by Seq. In the rest of this section we shall mainly be interested in the weak-* topology on $\ell_{1}(\mathrm{Seq})=c_{0}^{*}(\mathrm{Seq})$.

Definition 3.6. For each $s \in$ Seq we define a distinguished point $y_{s} \in \ell_{1}$ (Seq) by

$$
y_{s}(t)= \begin{cases}\#\left(t^{\prime}\right) & \text { if } t \subseteq s, \\ 0 & \text { otherwise. }\end{cases}
$$

Using the convention that sequences are to be identified with their Gödel numbers, we can write $y_{s}(t)=t^{\prime}$ if $t \subseteq s, 0$ otherwise. Note that, for all $s \in$ Seq, the sequence $\left\langle y_{s} \prec\langle n\rangle: n \in \mathbb{N}\right\rangle$, converges weak-* to $y_{s}$ in $\ell_{1}(\mathrm{Seq})$.

Definition 3.7. Given $s \in$ Seq we set

$$
Z_{s}=\left\{z \in \ell_{1}(\mathrm{Seq}) \mid \frac{1}{s^{\prime}} z(s)=\frac{1}{s} \sum_{m \in \mathbb{N}} z\left(s^{\curvearrowright}\langle m\rangle\right)\right\} .
$$

If $S \subseteq$ Seq, let $Z_{S}=\bigcap_{s \in S} Z_{s}$. Note that $Z_{S}$ is a norm closed subspace of $\ell_{1}$ (Seq). Note also that $y_{s} \in Z_{S}$ if and only if $s \notin S$.

The next two lemmas imply that, for any well-founded tree $T, Z_{T}$ is weak-* dense in $\ell_{1}$ (Seq).

Lemma 3.8. If $T$ is a well-founded tree, then $y_{s} \in Z_{T}^{\left(h_{T}(s)+1\right)}$ for all $s \in$ Seq.

Proof. We proceed by induction on $h_{T}(s)$. If $h_{T}(s)=-1$ then $s \notin T$ and $y_{s} \in$ $Z_{T}=Z_{T}^{(0)}$. Suppose now that $h_{T}(s)=\alpha \geq 0$, and that the theorem holds for all $t$ such that $h_{T}(t)<\alpha$. Then for each $n \in \mathbb{N}, h_{T}\left(s^{\frown}\langle n\rangle\right)<\alpha$, so $y_{s^{\wedge}\langle n\rangle} \in Z_{T}^{(\alpha)}$ for all $n \in \mathbb{N}$. Since $y_{s}$ is the weak-* limit of the sequence $\left\langle y_{s} \prec\langle n\rangle: n \in \mathbb{N}\right\rangle$, it follows that $y_{s} \in Z_{T}^{(\alpha+1)}$, as desired. 
Lemma 3.9. If $T$ is a well-founded tree, then $Z_{T}$ is weak-* dense in $\ell_{1}(\mathrm{Seq})$; in fact, $Z_{T}^{(h(T)+1)}=\ell_{1}$ (Seq).

Proof. Let $z \in \ell_{1}$ (Seq) be given. We can write $z=\sum_{s \in \operatorname{Seq}} z(s) \chi_{s}$, where $\chi_{s} \in$ $\ell_{1}$ (Seq) is the characteristic function of $\{s\}$, i.e., $\chi_{s}(t)=1$ if $t=s, 0$ otherwise. Note that if $s \neq\langle\rangle$ then $y_{s}-y_{s^{\prime}}=s^{\prime} \chi_{s}$, whereas $y_{\langle\rangle}=\chi_{\langle\rangle}$. Also, by the previous lemma, if $s \neq\langle\rangle$ then $y_{s} \in Z_{T}^{(h(T))}$, so if $\operatorname{lh}(s)>1$ then $\chi_{s} \in Z_{T}^{(h(T))}$. Since $z$ is an absolutely summable series, we have

$$
\begin{aligned}
\sum_{s \in \text { Seq }} z(s) \chi_{s} & =z(\langle\rangle) y_{\langle\rangle}+\sum_{m \in \mathbb{N}} z(\langle m\rangle) \chi_{\langle m\rangle}+\sum_{\substack{s \in \operatorname{Seq} \\
\operatorname{lh}(s)>1}} z(s) \chi_{s} \\
& =z(\langle\rangle) y_{\langle\rangle}+\sum_{m \in \mathbb{N}} z(\langle m\rangle)\left(y_{\langle m\rangle}-y_{\langle\rangle}\right)+\sum_{\operatorname{lh}(s)>1} z(s) \chi_{s} \\
& =\left(z(\langle\rangle)-\sum_{m \in \mathbb{N}} z(\langle m\rangle)\right) y_{\langle\rangle}+\sum_{m \in \mathbb{N}} z(\langle m\rangle) y_{\langle m\rangle}+\sum_{\operatorname{lh}(s)>1} z(s) \chi_{s} .
\end{aligned}
$$

Now, $z(\langle\rangle)-\sum_{m \in \mathbb{N}} z(\langle m\rangle)$ is just a real number since $z$ is absolutely summable, and $y_{\langle\rangle}$is the weak-* limit of the sequence $\left\langle y_{\langle m\rangle}: m \in \mathbb{N}\right\rangle$, so the first term in this last sum is in $Z_{T}^{(h(T)+1)}$. Likewise $\sum_{m \in \mathbb{N}} z(\langle m\rangle) y_{\langle m\rangle}+\sum_{\mathrm{lh}(s)>1} z(s) \chi_{s}$, viewed as a series in $\ell_{1}(\mathrm{Seq})$, converges in norm, and hence converges weak-*; since the functionals $y_{\langle m\rangle}$ and $\chi_{s}$ are in $Z_{T}^{(h(T))}$ for all $m \in \mathbb{N}$ and all $s \in \operatorname{Seq}$ with $\operatorname{lh}(s)>1$, it, too, is the weak-* limit of a sequence from $Z_{T}^{(h(T))}$. Hence $z$ is the sum of two weak-* limits of sequences from $Z_{T}^{(h(T))}$, whence $z$ is in $Z_{T}^{(h(T)+1)}$ as desired.

Corollary 3.10. If $T$ is a well-founded tree, then $\operatorname{ord}\left(Z_{T}\right) \leq h(T)+1$.

Thus we have an upper bound on the closure ordinal of $Z_{T}$ in terms of the height of $T$. To get a lower bound, we use the following technical lemma, which gives us a handle on the growth of the spaces $Z_{T}^{(\alpha)}$.

Lemma 3.11. Suppose that $z_{k} \rightarrow z$ weak-* in $\ell_{1}(\mathrm{Seq})=c_{0}^{*}(\mathrm{Seq})$. Let $s \in$ Seq be given and suppose that $z_{k} \in Z_{s}$ for all $k \in \mathbb{N}$. A sufficient condition for $z \in Z_{s}$ is the existence of $M \in \mathbb{N}$ such that $z_{k} \in Z_{s}\langle m\rangle$ for all $k \in \mathbb{N}$ and all $m \geq M$.

Proof. Assume that the stated condition holds. Suppose for a contradiction that $z \notin Z_{s}$, say

$$
\left|\frac{1}{s^{\prime}} z(s)-\frac{1}{s} \sum_{m=0}^{\infty} z\left(s^{\wedge}\langle m\rangle\right)\right|>\varepsilon>0 .
$$

Then for all sufficiently large $M$ we have

$$
\left|\frac{1}{s^{\prime}} z(s)-\frac{1}{s} \sum_{m<M} z\left(s^{\frown}\langle m\rangle\right)\right|>\varepsilon .
$$

Fix such an $M$, with $z_{k} \in Z_{s^{-}\langle m\rangle}$ for all $k \in \mathbb{N}$ and all $m \geq M$ as well. Then for all sufficiently large $k$ we have

$$
\left|\frac{1}{s^{\prime}} z_{k}(s)-\frac{1}{s} \sum_{m<M} z_{k}\left(s^{\wedge}\langle m\rangle\right)\right|>\varepsilon,
$$


and hence

$$
\begin{aligned}
& \varepsilon<\left|\frac{1}{s} \sum_{m \geq M} z_{k}\left(s^{\wedge}\langle m\rangle\right)\right| \\
& \leq \frac{1}{s} \sum_{m \geq M}\left|z_{k}\left(s^{\curvearrowright}\langle m\rangle\right)\right| \\
& =\frac{1}{s} \sum_{m \geq M}\left|\frac{s}{s^{\frown}\langle m\rangle} \sum_{n=0}^{\infty} z_{k}\left(s^{\frown}\langle m, n\rangle\right)\right| \quad\left(\text { since } z_{k} \in Z_{s \frown\langle m\rangle} \text { for all } m \geq M\right) \\
& \leq \frac{1}{s \bumpeq\langle M\rangle} \sum_{m \geq M} \sum_{n=0}^{\infty}\left|z_{k}\left(s^{\frown}\langle m, n\rangle\right)\right| \\
& \leq \frac{\left\|z_{k}\right\|}{s^{\sim}\langle M\rangle},
\end{aligned}
$$

and hence $\left\|z_{k}\right\|>\left(s^{\frown}\langle M\rangle\right) \varepsilon$. Thus $\left\{\left\|z_{k}\right\| \mid k \in \mathbb{N}\right\}$ is unbounded, contradicting Corollary 2.3.

In particular, we have the following result.

Corollary 3.12. Let $S \subseteq$ Seq be such that, for each $s \in S, s^{\wedge}\langle m\rangle \in S$ for all but finitely many $m \in \mathbb{N}$. Then $Z_{S}$ is weak-* closed.

Proof. By Lemma $3.11 Z_{S}$ is weak-* sequentially closed. Hence by Corollary 2.11 $Z_{S}$ is weak-* closed.

In order to make use of this lemma, we consider a special class of trees known as smooth trees:

Definition 3.13. For $s, t \in$ Seq we say $s$ is majorized by $t$, written $s \lll t$, if $\operatorname{lh}(s)=$ $\operatorname{lh}(t)$ and $s(i) \leq t(i)$ for all $i<\operatorname{lh}(s)$. For any tree $T$, we define $T^{*}$ to be the upward closure of $T$ under majorization, i.e.,

$$
T^{*}=\{t \in \operatorname{Seq} \mid \exists s \in T(s \lll t)\} .
$$

A tree $T$ is said to be smooth if it is upward closed under majorization, i.e., $T^{*}=T$.

Lemma 3.14 (Marcone $[15,16])$. Let $T$ be a tree. Then $T$ is well-founded if and only if $T^{*}$ is well-founded, in which case $h(T)=h\left(T^{*}\right)$.

Proof. Note first that $T \subseteq T^{*}$, so if $T^{*}$ is well-founded then so is $T$. Conversely, suppose $T^{*}$ has a path $f$; let $T_{f}=\{s \in T \mid s \lll f[\operatorname{lh}(s)]\}$. Then $T_{f}$ is a finitelybranching subtree of $T$, and, since $f$ is a path through $T^{*}, T_{f}$ must be infinite. Hence by König's Lemma $T_{f}$ has a path, whence $T$ has a path.

Assuming $T$ and $T^{*}$ are well-founded, we obviously have $h(T) \leq h\left(T^{*}\right)$. For the opposite inequality, we claim that for all $s, h_{T^{*}}(s)=\max \left\{h_{T}(t) \mid t \lll s\right\}$. (Note that $\{t \mid t \ll s\}$ is a finite set, so we may take max rather than sup.) We prove the claim by induction on $h_{T^{*}}(s)$. If $h_{T^{*}}(s)=-1$ then $s \notin T^{*}$, so for any $t$ with $t \lll s$ 
we have $t \notin T$, whence $h_{T}(t)=-1$ for all such $t$. Otherwise $s \in T^{*}$ and we have

$$
\begin{aligned}
h_{T^{*}}(s) & =\sup \left\{h_{T^{*}}\left(s^{\frown}\langle n\rangle\right)+1 \mid n \in \mathbb{N}\right\} \\
& =\sup \left\{\max \left\{h_{T}\left(t^{\frown}\langle m\rangle\right)+1 \mid t \lll s, m \leq n\right\} \mid n \in \mathbb{N}\right\} \\
& =\sup \left\{h_{T}\left(t^{\frown}\langle n\rangle\right)+1 \mid t \lll s, n \in \mathbb{N}\right\} \\
& =\max \left\{\sup \left\{h_{T}\left(t^{\frown}\langle n\rangle\right)+1 \mid n \in \mathbb{N}\right\} \mid t \lll s\right\} \\
& =\max \left\{h_{T}(t) \mid t \lll s\right\} .
\end{aligned}
$$

This proves our claim. In particular $h\left(T^{*}\right)=h_{T^{*}}(\langle\rangle)=h_{T}(\langle\rangle)=h(T)$, and the proof of the lemma is complete.

Corollary 3.15. For any countable ordinal $\alpha$, there exists a smooth well-founded tree $T$ such that $h(T)=\alpha$.

Proof. This follows immediately from Theorem 3.4 and the previous lemma.

Lemma 3.16. If $T$ is a smooth tree, then $T^{(\alpha)}$ is smooth for all $\alpha$.

Proof. We proceed by induction on $\alpha$. For $\alpha=0$ there's nothing to prove. Assume $T^{(\alpha)}$ is smooth, and let $s \in T^{(\alpha+1)}$ be given. Suppose $s \lll t$; since $s \in T^{(\alpha+1)} \subseteq T^{(\alpha)}$ which is smooth, $t$ must be in $T^{(\alpha)}$; furthermore, since $s$ is an interior node of $T^{(\alpha)}$, there is an $m \in \mathbb{N}$ such that $s^{\frown}\langle m\rangle \in T^{(\alpha)}$. But $s^{\frown}\langle m\rangle \lll t^{\frown}\langle m\rangle$, so $t^{\frown}\langle m\rangle \in T^{(\alpha)}$, whence $t \in T^{(\alpha+1)}$. Finally, smoothness is clearly preserved under intersections, so the induction goes through at limit stages.

Lemma 3.17. Let $T$ be a smooth well-founded tree. Then for all $\alpha \leq h(T), Z_{T}^{(\alpha)} \subseteq$ $Z_{T^{(\alpha)}}$.

Proof. We proceed by induction on $\alpha$. If $\alpha=0$ there's nothing to prove. Assume $Z_{T}^{(\alpha)} \subseteq Z_{T^{(\alpha)}}$ and $\alpha<h(T)$, and let $z \in Z_{T}^{(\alpha+1)}$ be given. Then $z$ is the weak-* limit of some sequence $\left\langle z_{k} \mid k \in \mathbb{N}\right\rangle$ from $Z_{T}^{(\alpha)} \subseteq Z_{T^{(\alpha)}}$. Since $\alpha<h(T)$, we have that any $s \in T^{(\alpha+1)}$ is an interior node of $T^{(\alpha)}$, i.e., $s \in T^{(\alpha)}$ and $s^{\curlyvee}\langle M\rangle \in T^{(\alpha)}$ for some $M$. Since $T$ is smooth, so is $T^{(\alpha)}$, and hence $s^{\frown}\langle m\rangle \in T^{(\alpha)}$ for all $m \geq M$. Hence, for each $k \in \mathbb{N}$ we have $z_{k} \in Z_{s}$ and $z_{k} \in Z_{s \sim\langle m\rangle}$ for all $m \geq M$. By Lemma 3.11 it follows that $z \in Z_{s}$. Since $s$ is an arbitrary node in $T^{(\alpha+1)}$, we have $z \in Z_{T^{(\alpha+1)}}$. This shows that $Z_{T}^{(\alpha+1)} \subseteq Z_{T^{(\alpha+1)}}$. Finally, if $\delta$ is a limit ordinal $\leq h(T)$ and $Z_{T}^{(\alpha)} \subseteq Z_{T^{(\alpha)}}$ for all $\alpha<\delta$, it follows easily that $Z_{T^{(\alpha)}} \subseteq Z_{T^{(\delta)}}$ for all $\alpha<\delta$, whence $Z_{T}^{(\delta)} \subseteq Z_{T^{(\delta)}}$. This completes the proof.

Corollary 3.18. If $T$ is a smooth well-founded tree, then $\operatorname{ord}\left(Z_{T}\right)=h(T)+1$.

Proof. By Lemma 3.9 we have $Z_{T}^{\left(\operatorname{ord}\left(Z_{T}\right)\right)}=\ell_{1}($ Seq $)$ and $\operatorname{ord}\left(Z_{T}\right) \leq h(T)+1$. On the other hand, $T^{(h(T))}=\{\langle\rangle\}$, so $y_{\langle\rangle} \notin Z_{T^{(h(T))}}$; hence, by the previous lemma, $y_{\langle\rangle} \notin Z_{T}^{(h(T))}$, so in particular $Z_{T}^{(h(T))} \neq \ell_{1}(\mathrm{Seq})$, and hence $h(T)<\operatorname{ord}\left(Z_{T}\right)$. This completes the proof.

We now obtain the main result of this section, originally due to McGehee [18]:

Theorem 3.19. For any countable ordinal $\alpha$, there exists a weak-* dense subspace $Z$ of $\ell_{1}=c_{0}^{*}$ such that $\operatorname{ord}(Z)=\alpha+1$. 
Proof. Since Seq is a countably infinite set, we may identify $\ell_{1}=c_{0}^{*}$ with $\ell_{1}(\mathrm{Seq})=$ $c_{0}^{*}$ (Seq). By Corollary 3.15 let $T$ be a smooth well-founded tree of height $\alpha$. By Lemma $3.9 Z_{T}$ is weak-* dense in $\ell_{1}$, and by Corollary 3.18 we have $\operatorname{ord}\left(Z_{T}\right)=$ $h(T)+1=\alpha+1$.

Corollary 3.20. The ordinals which can occur as closure ordinals of subspaces of the dual of a separable Banach space are precisely the countable non-limit ordinals.

Proof. This is immediate from Theorems 2.13 and 3.19. The result is originally due to Sarason [19, 20, 21] and McGehee [18].

This settles the question of closure ordinals of subspaces of $\ell_{1}=c_{0}^{*}$, but what about $\ell_{2}$ ? It turns out that the answer is much simpler (see Corollary 3.23 below).

Theorem 3.21. Let $X$ be a Banach space, and let $C \subseteq X$ be convex. Then for any $x \in X$, the following conditions are equivalent:

1. $x$ is in the weak closure of $C$.

2. $x$ is in the norm closure of $C$.

3. $x$ is the norm limit of a sequence of points in $C$.

4. $x$ is the weak limit of a sequence of points in $C$.

Proof. The equivalence of (1) and (2) follows from the well-known fact (Theorem V.3.13 in [11]) that a convex set is weakly closed if and only if it is norm closed. The implications $(2) \Rightarrow(3) \Rightarrow(4) \Rightarrow(1)$ are all trivial.

Corollary 3.22. If $X$ is a reflexive Banach space and $C \subseteq X^{*}$ is convex, then the weak-* sequential closure ordinal of $C$ is 0 if $C$ is weak-* closed, and is 1 otherwise.

Proof. If $X$ is reflexive then so is $X^{*}$ (see Corollary II.3.24 in [11]), and hence the weak and weak-* topologies on $X^{*}$ coincide. The result now follows immediately from Theorem 3.21.

We would like to thank Howard Becker for pointing this out to us.

Corollary 3.23. If $C \subseteq \ell_{2}$ is convex, then the weak-* (i.e., weak) sequential closure ordinal of $C$ is 0 if $C$ is weak-* (i.e., weakly) closed, 1 otherwise.

We do not know whether the closure ordinal of a convex set in the dual of a separable Banach space can be a limit ordinal.

\section{The WeAK-* TOPOlOGY IN SUBSystems of $Z_{2}$}

The language of second order arithmetic consists of number variables $m, n, \ldots$, set variables $X, Y, \ldots$, primitives $+, \cdot, 0,1,=, \in$, and logical operations including number quantifiers and set quantifiers. By second order arithmetic (sometimes called $Z_{2}$ ) we mean the theory consisting of classical logic plus certain basic arithmetical axioms plus the induction scheme

$$
(\varphi(0) \wedge \forall n(\varphi(n) \rightarrow \varphi(n+1))) \rightarrow \forall n \varphi(n)
$$

plus the comprehension scheme

$$
\exists W \forall n(n \in W \leftrightarrow \varphi(n))
$$

where $\varphi(n)$ is an arbitrary formula of the language of second order arithmetic. In the comprehension scheme it is assumed that the set variable $W$ does not occur freely in $\varphi(n)$. All of the subsystems of second order arithmetic that we shall 
consider employ classical logic and include the basic arithmetical axioms and the restricted induction axiom

$$
(0 \in W \wedge \forall n(n \in W \rightarrow n+1 \in W)) \rightarrow \forall n(n \in W) .
$$

Two of the most important subsystems of second order arithmetic are ACA $_{0}$ and $\Pi_{1}^{1}-C A_{0}$. A formula of the language of second order arithmetic is said to be arithmetical if it contains no set quantifiers. The axioms of $\mathrm{ACA}_{0}$ consist of the basic arithmetical axioms, the restricted induction axiom, and arithmetical comprehension, i.e., the comprehension scheme for formulas $\varphi(n)$ which are arithmetical. A $\Pi_{1}^{1}$ formula is one of the form $\forall W \theta$ where $\theta$ is arithmetical. The axioms of $\Pi_{1}^{1}-C_{0}$ consist of the axioms of $A C A_{0}$ plus $\Pi_{1}^{1}$ comprehension. Obviously $\Pi_{1}^{1}-C_{0}$ is much stronger than $A C A_{0}$. Three other very important subsystems of second order arithmetic are $R C A_{0}$ and $W K L_{0}$, both of which are weaker than $A C A_{0}$, and $A T R_{0}$, which is intermediate between $A C A_{0}$ and $\Pi_{1}^{1}-C A_{0}$. For background material on subsystems of second order arithmetic, we refer the reader to $[12,6,7,23]$.

The purpose of this section is to show how some fundamental results concerning separable Banach spaces and the weak-* topology can be developed formally within $\mathrm{ACA}_{0}$ and weaker systems. In particular, we show that a version of the Krein-Šmulian theorem is provable in $\mathrm{ACA}_{0}$. Our approach for the development of separable Banach space theory within subsystems of second order arithmetic follows that of Brown and Simpson [6, 4, 5, 7, 23]; see also the paper of Shioji and Tanaka [22].

Definition $4.1\left(\mathrm{RCA}_{0}\right)$. A (code for a) complete separable metric space $\widehat{A}$ is defined to be a set $A \subseteq \mathbb{N}$ together with a function $d: A \times A \rightarrow \mathbb{R}$ such that, for all $a, b, c \in A$,

1. $d(a, a)=0$,

2. $d(a, b)=d(b, a)$, and

3. $d(a, c) \leq d(a, b)+d(b, c)$.

A (code for a) point of $\widehat{A}$ is defined to be a sequence $\left\langle a_{n} \mid n \in \mathbb{N}\right\rangle$ of elements of $A$ such that $\forall m \forall n\left(m<n \longrightarrow d\left(a_{m}, a_{n}\right) \leq 1 / 2^{m}\right)$. Although $\widehat{A}$ does not formally exist as a set within $\mathrm{RCA}_{0}$, we use notations such as $x \in \widehat{A}$ to mean that $x$ is a point of $\widehat{A}$, etc. We then straightforwardly extend our definitions of $=$ and $d$ to $\widehat{A}$ in such a way that $\langle\widehat{A}, d\rangle$ is a complete metric space, with $A$ dense in $\widehat{A}$.

Definition $4.2\left(\mathrm{RCA}_{0}\right)$. Let $\widehat{A}$ be a complete separable metric space as defined above. A (code for an) open set in $\widehat{A}$ is defined to be a sequence of ordered pairs $U=\left\langle\left(a_{i}, q_{i}\right) \mid i \in \mathbb{N}\right\rangle, a_{i} \in A, q_{i} \in \mathbb{Q}$. We write $x \in U$ to mean that $x \in \widehat{A}$ and $d\left(x, a_{i}\right)<q_{i}$ for some $i \in \mathbb{N}$. A closed set in $\widehat{A}$ is defined to be the complement of an open set.

Building on the definitions above within $\mathrm{RCA}_{0}$, one can define corresponding notions of continuous function from one complete separable metric space into another, etc. On this basis, one can prove within $\mathrm{ACA}_{0}$ or $\mathrm{WKL}_{0}$ or $\mathrm{RCA}_{0}$ many fundamental results about the topology of complete separable metric spaces. For details, see [23]. In particular, one can prove within $\mathrm{WKL}_{0}$ (see Chapter IV of [23]) the Heine-Borel covering lemma ("if $C$ is compact then any covering of $C$ by a sequence of open sets has a finite subcovering"), using the following $\mathrm{RCA}_{0}$ notion of compactness: 
Definition $4.3\left(\mathrm{RCA}_{0}\right)$. Let $C$ be a closed set in a complete separable metric space $\widehat{A}$. We say that $C$ is compact if there exists a countable sequence of finite sequences of points $\left\langle\left\langle x_{n i} \mid i \leq k_{n}\right\rangle \mid n \in \mathbb{N}\right\rangle$ in $\widehat{A}$ such that for all $x \in C$ and all $n \in \mathbb{N}$ there exists $i \leq k_{n}$ such that $d\left(x, x_{n i}\right)<1 / 2^{n}$.

In the same setting, there is a useful version of the Tychonoff product theorem, and one can prove within $\mathrm{RCA}_{0}$ the compactness of the product space $\prod_{n \in \mathbb{N}}\left[a_{n}, b_{n}\right]$, where $\left\langle\left[a_{n}, b_{n}\right] \mid n \in \mathbb{N}\right\rangle$ is any sequence of closed bounded intervals. For details, see Chapter IV of [23].

We now turn to our development of separable Banach space theory within $\mathrm{RCA}_{0}$ and $\mathrm{ACA}_{0}$.

Definition $4.4\left(\mathrm{RCA}_{0}\right)$. A (code for a) separable Banach space consists of a countable set $A \subseteq \mathbb{N}$ together with operations $+: A \times A \rightarrow A,-: A \times A \rightarrow A$, and $\cdot: \mathbb{Q} \times A \rightarrow A$ and a distinguished element $0 \in A$ such that $\langle A,+,-, \cdot, 0\rangle$ forms a countable vector space over the rational field $\mathbb{Q}$, together with a function \|\|$: A \rightarrow \mathbb{R}$ satisfying

1. $\|q a\|=|q|\|a\|$ for all $a \in A$ and $q \in \mathbb{Q}$, and

2. $\|a+b\| \leq\|a\|+\|b\|$ for all $a, b \in A$.

In other words, a code for a separable Banach space $\widehat{A}$ is a countable pseudo-normed vector space $A$ over $\mathbb{Q}$. Note that $\widehat{A}$ is a complete separable metric space under $d(a, b)=\|a-b\|$. Thus a point of the separable Banach space $\widehat{A}$ is by definition a sequence $\left\langle a_{n} \mid n \in \mathbb{N}\right\rangle$ such that $\forall m \forall n\left(m<n \longrightarrow\left\|a_{m}-a_{n}\right\|<1 / 2^{m}\right)$.

Definition $4.5\left(\mathrm{RCA}_{0}\right)$. Let $X=\widehat{A}$ and $Y=\widehat{B}$ be separable Banach spaces. A (code for a) bounded linear operator $F: X \rightarrow Y$ is a linear mapping $F: A \rightarrow \widehat{B}$ such that, for some $0 \leq r<\infty,\|F\| \leq r$, i.e., $\|F(a)\| \leq r\|a\|$ for all $a \in A$. If $x=\left\langle a_{n} \mid n \in \mathbb{N}\right\rangle$ is a point of $X=\widehat{A}$, we write $F(x)=\lim _{n} F\left(a_{n}\right)$.

It can be proved in $\mathrm{RCA}_{0}[6,4,23]$ that bounded linear operators $F: X \rightarrow Y$ are identifiable with continuous linear mappings from $X$ into $Y$. Also within $\mathrm{RCA}_{0}$ one can prove a useful version of the Banach-Steinhaus theorem:

Theorem 4.6. The following is provable in $\mathrm{RCA}_{0}$. Given separable Banach spaces $X$ and $Y$ and a sequence of bounded linear operators $F_{n}: X \rightarrow Y, n \in \mathbb{N}$, if $\left\{\left\|F_{n}(x)\right\| \mid n \in \mathbb{N}\right\}$ is bounded for all $x \in X$, then there exists $r<\infty$ such that $\left\|F_{n}\right\| \leq r$ for all $n \in \mathbb{N}$.

Proof. See $[4,7,23]$.

Definition 4.7 $\left(\mathrm{RCA}_{0}\right)$. Let $X$ be a separable Banach space. A bounded linear functional on $X$ is a bounded linear operator $f: X \rightarrow \mathbb{R}$. We write $f \in X^{*}$ to mean that $f$ is a bounded linear functional on $X$. For $0 \leq r<\infty$, we write $f \in B_{r}\left(X^{*}\right)$ to mean that $f \in X^{*}$ and $\|f\| \leq r$.

Note that $X^{*}$ and $B_{r}\left(X^{*}\right)$ do not formally exist as sets within $\mathrm{RCA}_{0}$. We identify the functionals in $B_{r}\left(X^{*}\right)$ in the obvious way with the points of a certain closed set in the compact metric space $\prod_{a \in A}[-r\|a\|, r\|a\|]$, where $X=\widehat{A}$. Thus the compactness of $B_{r}\left(X^{*}\right)$ is provable in $\mathrm{RCA}_{0}$. This version of the Banach-Alaoglu theorem turns out to be very useful for the development of separable Banach space theory within $\mathrm{WKL}_{0}$. See $[6,22]$ and Chapter IV of [23] and Brown's discussion of the "Alaoglu ball" [4]. In particular we have: 
Theorem 4.8. The following version of the Hahn-Banach theorem is provable in $\mathrm{WKL}_{0}$. Let $X$ be a separable Banach space and let $Y$ be a subspace of $X$. If $g: Y \rightarrow \mathbb{R}$ is a bounded linear functional with $\|g\| \leq r$, then there exists a bounded linear functional $f: X \rightarrow \mathbb{R}$ such that $\|f\| \leq r$ and $f$ extends $g$, i.e., $f(x)=g(x)$ for all $x \in Y$.

Proof. The literature contains two proofs of this result. A direct proof is in [6]. An indirect proof via a $\mathrm{WKL}_{0}$ version of the Markov-Kakutani fixed point theorem is in [22] and Chapter IV of [23].

Theorem 4.9. The following extension of the Hahn-Banach Theorem is provable in $\mathrm{WKL}_{0}$. Let $X$ be a separable Banach space. Let $p: X \rightarrow \mathbb{R}$ be a continuous function such that $p(r x)=r p(x)$ and $p(x+y) \leq p(x)+p(y)$ for all $r \geq 0$ and $x, y \in X$. Let $Y$ be a subspace of $X$ and let $g: Y \rightarrow \mathbb{R}$ be a bounded linear functional such that $g(x) \leq p(x)$ for all $x \in Y$. Then there exists a bounded linear functional $f: X \rightarrow \mathbb{R}$ such that $f$ extends $g$ and $f(x) \leq p(x)$ for all $x \in X$.

Proof. Either of the cited proofs of Theorem 4.8 can be straightforwardly adapted to prove this more general result. See also Theorem 4.2 of [4].

For use later in this section, we note that the following separation principle holds in $\mathrm{ACA}_{0}$ :

Lemma 4.10. The following is provable in $\mathrm{ACA}_{0}$. Let $X$ be a separable Banach space. Let $Z$ be a countable set in $X$ such that $\|x\| \geq 1$ for all $x$ in the convex hull of $Z$. Then there exists $f \in B_{1}\left(X^{*}\right)$ such that $f(x) \geq 1$ for all $x$ in the convex hull of $Z$.

Proof. Put

$$
W=\left\{\sum_{i<n} q_{i} z_{i} \mid n \in \mathbb{N}, q_{i} \in \mathbb{Q} \cap[0,1], \sum_{i<n} q_{i}=1, z_{i} \in Z\right\} .
$$

Note that $\|w\| \geq 1$ for all $w \in W$. Fix $x_{0} \in W$. By arithmetical comprehension, there is a continuous function $p: X \rightarrow \mathbb{R}$ defined by

$$
p(x)=\inf \left\{c \in \mathbb{Q} \mid c>0 \wedge \exists w \in W\left(\left\|(x / c)+w-x_{0}\right\| \leq 1\right)\right\} .
$$

Then $p$ satisfies the following:

1. $p\left(x_{0}\right) \geq 1$.

2. $0 \leq p(x) \leq\|x\|$ for all $x \in X$.

3. $p(r x)=r p(x)$ for all $r \geq 0$ and $x \in X$.

4. $p(x+y) \leq p(x)+p(y)$ for all $x, y \in X$.

Some remarks: if property 1 failed, then there would be $w \in W$ and a rational $c<1$ such that $\left\|\left(x_{0} / c\right)+w-x_{0}\right\| \leq 1$, whence $\left\|(1-c) x_{0}+c w\right\| \leq c<1$, contrary to $(1-c) x_{0}+c w$ being in $W$. Properties 2 and 3 are easily verified. As for property 4 , given $c>p(x)+p(y)$, write $c=a+b$ where $a>p(x)$ and $b>p(y)$. Then there exist $w_{1}$ and $w_{2}$ in $W$ such that $\left\|(x / a)+w_{1}-x_{0}\right\| \leq 1$ and $\left\|(y / b)+w_{2}-x_{0}\right\| \leq 1$. Then

$$
\begin{aligned}
\frac{x+y}{a+b} & +\frac{a}{a+b} w_{1}+\frac{b}{a+b} w_{2}-x_{0} \\
= & \left(\frac{a}{a+b}\right)\left(\frac{x}{a}+w_{1}-x_{0}\right)+\left(\frac{b}{a+b}\right)\left(\frac{y}{b}+w_{2}-x_{0}\right)
\end{aligned}
$$


SO

$$
\left\|\frac{x+y}{a+b}+\frac{a}{a+b} w_{1}+\frac{b}{a+b} w_{2}-x_{0}\right\| \leq \frac{a}{a+b}+\frac{b}{a+b}=1,
$$

whence $p(x+y) \leq a+b=c$.

Let $Y$ be the subspace of $X$ generated by $x_{0}$, i.e., $Y=\mathbb{R} x_{0}$. Define $g: Y \rightarrow \mathbb{R}$ by $g\left(r x_{0}\right)=r p\left(x_{0}\right)$. Then $g$ is a bounded linear functional on $Y$. Moreover, if $r \geq 0$ then $g\left(r x_{0}\right)=r p\left(x_{0}\right)=p\left(r x_{0}\right)$, and if $r<0$ then $g\left(r x_{0}\right)=r p\left(x_{0}\right) \leq 0 \leq p\left(r x_{0}\right)$, so $g \leq p$ on $Y$. Thus, by our extended Hahn-Banach theorem 4.9 in $\mathrm{WKL}_{0}$, we can extend $g$ to a bounded linear functional $f: X \rightarrow \mathbb{R}$ such that $f \leq p$ on $X$.

Let $w \in W$ be given, and suppose $y$ is such that $\|y\| \leq 1$. Then

$$
\left\|\left(y-w+x_{0}\right)+w-x_{0}\right\|=\|y\| \leq 1,
$$

whence $f\left(y-w+x_{0}\right) \leq p\left(y-w+x_{0}\right) \leq 1$ by definition of $p$. But $f\left(y-w+x_{0}\right)=$ $f(y)-f(w)+f\left(x_{0}\right)$ and $f\left(x_{0}\right) \geq 1$, so $f(y) \leq f(w)$. Replacing $f$ by $f /\|f\|$, we see that $f \in B_{1}\left(X^{*}\right)$ and $f(x) \geq 1$ for all $x$ in the convex hull of $Z$. This completes the proof.

We conjecture that this separation principle is actually provable in $\mathrm{WKL}_{0}$ and not only in $\mathrm{ACA}_{0}$.

We now begin our treatment of the weak-* topology within RCA . We start by introducing an $\mathrm{RCA}_{0}$ version of the bounded-weak-* topology:

Definition $4.11\left(\mathrm{RCA}_{0}\right)$. A (code for a) bounded-weak-*-closed set $C$ in $X^{*}$ is defined to be a sequence of (codes for) closed sets $C_{n} \subseteq B_{n}\left(X^{*}\right), n \in \mathbb{N}$, such that

$$
\forall m \forall n\left(m<n \longrightarrow C_{m}=B_{m}\left(X^{*}\right) \cap C_{n}\right) .
$$

We write $x^{*} \in C$ to mean $\exists n\left(x^{*} \in C_{n}\right)$, or equivalently $\forall n\left(n>\left\|x^{*}\right\| \rightarrow x^{*} \in C_{n}\right)$. A bounded-weak-*-open set in $X^{*}$ is defined to be the complement of a boundedweak-*-closed set in $X^{*}$.

The next lemma formalizes within $\mathrm{ACA}_{0}$ a well-known fact (see Lemma V.5.4 in [11]): there is a bounded-weak-* neighborhood basis of 0 in $X^{*}$ consisting of the polars of sequences converging to 0 in $X$.

Lemma 4.12. The following is provable in $\mathrm{ACA}_{0}$. Let $X$ be a separable Banach space. If $\left\langle x_{n} \mid n \in \mathbb{N}\right\rangle$ is a sequence of points in $X$ such that $x_{n} \rightarrow 0$, then

$$
\left\{x^{*} \in X^{*}|\forall n| x^{*}\left(x_{n}\right) \mid<1\right\}
$$

contains 0 and is bounded-weak-*-open in $X^{*}$. Conversely, if $U$ is a bounded-weak*-open set in $X^{*}$ containing 0 , then we can find a sequence of points $\left\langle x_{n} \mid n \in \mathbb{N}\right\rangle$ in $X$ such that $x_{n} \rightarrow 0$ and $\left\{x^{*} \in X^{*}|\forall n| x^{*}\left(x_{n}\right) \mid \leq 1\right\} \subseteq U$.

Proof. Reasoning in $\mathrm{ACA}_{0}$, let $\left\langle x_{n} \mid n \in \mathbb{N}\right\rangle$ be a sequence of points in $X$ such that $\lim _{n} x_{n}=0$. By arithmetical comprehension, there exists a sequence of integers $N_{m}, m \in \mathbb{N}$, such that $\left\|x_{n}\right\|<1 / m$ for all $m \in \mathbb{N}$ and $n \geq N_{m}$. Using the sequence $\left\langle N_{m} \mid m \in \mathbb{N}\right\rangle$ as a parameter, we can define a sequence of closed sets $C_{m} \subseteq B_{m}\left(X^{*}\right), m \in \mathbb{N}$, by

$$
C_{m}=\left\{x^{*} \in B_{m}\left(X^{*}\right) \mid \exists n<N_{m}\left(\left|x^{*}\left(x_{n}\right)\right| \geq 1\right)\right\} .
$$

It is easy to verify that

$$
C_{m}=\left\{x^{*} \in B_{m}\left(X^{*}\right)|\exists n| x^{*}\left(x_{n}\right) \mid \geq 1\right\}
$$


and hence $C_{k}=C_{m} \cap B_{k}\left(X^{*}\right)$ for all $k<m$. Thus by Definition 4.11 we have a bounded-weak- $*$-closed set $C=\bigcup_{m \in \mathbb{N}} C_{m}$, and clearly

$$
X^{*} \backslash C=\left\{x^{*} \in X^{*}|\forall n| x^{*}\left(x_{n}\right) \mid<1\right\} .
$$

This shows that $\left\{x^{*} \in X^{*}|\forall n| x^{*}\left(x_{n}\right) \mid<1\right\}$ is bounded-weak-*-open in $X^{*}$.

The proof of the converse will be carried out in $\mathrm{WKL}_{0}$. Let $U$ be a boundedweak-*-open set in $X^{*}$ containing 0 . Then $C=X^{*} \backslash U$ is a bounded-weak-*-closed set with $0 \notin C$. For any countable set $S \subseteq X$ let $S^{o}$ be the polar of $S$, i.e.,

$$
S^{o}=\left\{x^{*} \in X^{*}|\forall x \in S| x^{*}(x) \mid \leq 1\right\} .
$$

To complete the proof, we need to construct a sequence of points $\left\langle x_{k} \mid k \in \mathbb{N}\right\rangle$ in $X$ such that $\lim _{k} x_{k}=0$ and $\left\{x_{k} \mid k \in \mathbb{N}\right\}^{\circ} \cap C=\varnothing$.

Let $X=\widehat{A}$ where $A$ is a countable dense set in $X$. Put $A_{0}=A$ and, for each $n \geq 1, A_{n}=\{a \in A \mid\|a\|<1 / n\}$. Claim: for any $n \in \mathbb{N}$ and any countable set $S \subseteq X$, if $S^{o} \cap B_{n}\left(X^{*}\right) \cap C=\varnothing$ then there exists a finite set $F \subset A_{n}$ such that $(S \cup F)^{o} \cap B_{n+1}\left(X^{*}\right) \cap C=\varnothing$. If such an $F$ does not exist, then for all finite sets $F \subset A_{n}$ we would have $(S \cup F)^{o} \cap B_{n+1}\left(X^{*}\right) \cap C \neq \varnothing$, so by the Heine-Borel covering property of the compact set $B_{n+1}\left(X^{*}\right)$ it would follow that

$$
\left(S \cup A_{n}\right)^{o} \cap B_{n+1}\left(X^{*}\right) \cap C \neq \varnothing .
$$

But $\left(S \cup A_{n}\right)^{o}=S^{o} \cap A_{n}^{o}=S^{o} \cap B_{n}\left(X^{*}\right)$, and hence $S^{o} \cap B_{n}\left(X^{*}\right) \cap C \neq \varnothing$, a contradiction. This proves the claim.

Within $\mathrm{WKL}_{0}$, we can apply the claim above repeatedly starting with $F_{0}=\varnothing$ to obtain a sequence of finite sets $F_{n+1} \subseteq A_{n}, n \in \mathbb{N}$, such that

$$
\left(F_{0} \cup \ldots \cup F_{n}\right)^{o} \cap B_{n}\left(X^{*}\right) \cap C=\varnothing
$$

for all $n \in \mathbb{N}$. The construction can be carried out effectively within $\mathrm{WKL}_{0}$ because, by Lemma 5.8 of [3], the predicates $F \subset A_{n}$ and $F^{o} \cap B_{n}\left(X^{*}\right) \cap C=\varnothing$ are provably in $\mathrm{WKL}_{0}$ equivalent to $\Sigma_{1}^{0}$ formulas. Thus the existence of a sequence of finite sets $\left\langle F_{n} \mid n \in \mathbb{N}\right\rangle$ with the mentioned properties is provable in $\mathrm{WKL}_{0}$.

Letting $\left\langle x_{k} \mid k \in \mathbb{N}\right\rangle$ be an enumeration without repetition of $\bigcup_{n \in \mathbb{N}} F_{n}$, it is clear that $x_{k} \rightarrow 0$ in $X$ and that $\left\{x_{k} \mid k \in \mathbb{N}\right\}^{\circ} \cap C=\varnothing$. This completes the proof.

We now introduce our $\mathrm{RCA}_{0}$ version of the weak-* topology.

Definition $4.13\left(\mathrm{RCA}_{0}\right)$. A weak-*-open set in $X^{*}$ is defined to be a boundedweak-*-open set $U$ in $X^{*}$ such that for all $x_{0}^{*} \in U$ there exists a finite sequence of points $x_{0}, \ldots, x_{n-1} \in X$ such that

$$
\left\{x^{*} \in X^{*} \mid \forall k<n\left(\left|x^{*}\left(x_{k}\right)-x_{0}^{*}\left(x_{k}\right)\right| \leq 1\right)\right\} \subseteq U .
$$

A weak-*-closed set in $X^{*}$ is defined to be the complement of a weak-*-open set in $X^{*}$.

According to the previous definition, we have trivially in $\mathrm{RCA}_{0}$ that any weak*-closed set is bounded-weak-*-closed. In $\mathrm{ACA}_{0}$ we have following version of the Krein-Šmulian theorem:

Theorem 4.14. The following is provable in $\mathrm{ACA}_{0}$. Let $X$ be a separable Banach space. Suppose that $C \subseteq X^{*}$ is convex and bounded-weak-*-closed. Then $C$ is weak-*-closed. 
Proof. Let $x_{0}^{*} \notin C$ be given. Then $C-x_{0}^{*}$ is also bounded-weak-*-closed and convex, and $0 \notin C-x_{0}^{*}$. Thus $\left(C-x_{0}^{*}\right) \cap B_{n}\left(X^{*}\right)$ is a closed subset of $B_{n}\left(X^{*}\right)$ for each $n \in \mathbb{N}$. Since $B_{n}\left(X^{*}\right)$ is compact, in $\mathrm{ACA}_{0}$ there exists a countable dense subset $D_{n} \subset\left(C-x_{0}^{*}\right) \cap B_{n}\left(X^{*}\right)$ for each $n$ (see Theorem 3.2 in [5]). We want to find a weak-*-open set $N$ containing 0 in $X^{*}$ which is disjoint from $C-x_{0}^{*}$, as this will show that $x_{0}^{*}+N$ is disjoint from $C$. Let $U$ be a bounded-weak-*-open set containing 0 which is disjoint from $C-x_{0}^{*}$. By Lemma 4.12, there is a sequence $\left\{x_{n} \mid n \in \mathbb{N}\right\}$ such that $x_{n} \rightarrow 0$ and $\left\{x^{*} \in X^{*}|\forall n| x^{*}\left(x_{n}\right) \mid \leq 1\right\} \subseteq U$.

Now, for each $m \in \mathbb{N}$, define a function $T_{m}: B_{m}\left(X^{*}\right) \rightarrow c_{0}$ by $T_{m}\left(x^{*}\right)=$ $\left\langle x^{*}\left(x_{n}\right) \mid n \in \mathbb{N}\right\rangle$. These form a sequence of compatible functions, i.e., if $m<n$ then $T_{n} \mid B_{m}\left(X^{*}\right)=T_{m}$. Thus we can define a function $T: X^{*} \rightarrow c_{0}$ by $T\left(x^{*}\right)=T_{m}\left(x^{*}\right)$ where $x^{*} \in B_{m}\left(X^{*}\right)$. Notice that $T$ is linear, and $\left\|T\left(x^{*}\right)\right\| \leq 1$ implies $x^{*} \in U$ for all $x^{*} \in X^{*}$. Let $D$ be an enumeration of the dense sets $D_{n} \subset\left(C-x_{0}^{*}\right) \cap B_{n}\left(X^{*}\right)$, and let $E=T(D)$ (which exists by arithmetical comprehension); then $E$ is a countable subset of $c_{0}$, and $\|x\|>1$ for all $x$ in the convex hull of $E$. By Lemma 4.10 there exists $f \in B_{1}\left(c_{0}^{*}\right)=B_{1}\left(\ell_{1}\right)$ such that $f(x) \geq 1$ for all $x$ in the convex hull of $E$. Write $f=\left\langle\alpha_{n}: n \in \mathbb{N}\right\rangle \in \ell_{1}$.

Let $x=\sum_{n \in \mathbb{N}} \alpha_{n} x_{n}$; note that $\left\{x^{*} \in X^{*}|| x^{*}(x) \mid<1\right\}$ is weak-*-open and contains 0. Also, if $y^{*} \in C-x_{0}^{*}$ then $y^{*} \in B_{m}\left(X^{*}\right)$ for some $m$. Thus $\left|y^{*}(x)\right|=$ $\left|\sum_{n \in \mathbb{N}} \alpha_{n} y^{*}\left(x_{n}\right)\right|=\left|f\left(T\left(y^{*}\right)\right)\right|=\left|f\left(T_{m}\left(y^{*}\right)\right)\right| \geq 1$, since $D_{m}$ is dense in $B_{m}\left(X^{*}\right)$ and $f$ and $T_{m}$ are continuous. So let $N=\left\{x^{*} \in X^{*}|| x^{*}(2 x) \mid<1\right\}=\left\{x^{*} \in X^{*} \mid\right.$ $\left.\left|x^{*}(x)\right|<1 / 2\right\}$; then $N$ is weak-*-open, contains 0 , and is disjoint from $C-x_{0}^{*}$. This completes the proof.

Specializing to subspaces of $X^{*}$ (see also Corollary 2.8 above), we obtain:

Corollary 4.15. The following is provable in $\mathrm{ACA}_{0}$. Let $X$ be a separable Banach space. Let $C$ be a closed set in $B_{1}\left(X^{*}\right)$ such that $C=B_{1}\left(X^{*}\right) \cap \operatorname{span}(C)$, where

$$
\operatorname{span}(C)=\left\{\sum_{i=0}^{n} a_{i} x_{i}^{*} \mid a_{i} \in \mathbb{R}, x_{i}^{*} \in C, n \in \mathbb{N}\right\} .
$$

Then $\operatorname{span}(C)$ is a weak-*-closed subspace of $X^{*}$.

Proof. This follows easily from the previous theorem. See also the proof of Corollary 2.8 .

\section{Proof of the Main Result}

In this section we show that a rather strong set existence axiom, $\Pi_{1}^{1}$ comprehension, is needed to prove a very elementary (indeed trivial-sounding) fact of separable Banach space theory. Namely, $\Pi_{1}^{1}-C_{A}$ is equivalent over $A C A_{0}$ to the following statement:

Given a separable Banach space $X$ and a countable set

$Y \subset X^{*}$, there is a smallest weak-*-closed subspace $C$ of $X^{*}$ such that $Y \subseteq C$.

This equivalence is the content of our main result, Theorem 5.6 below.

The forward direction is the assertion that $(S)$ is provable in $\Pi_{1}^{1}-C A_{0}$. This will be obtained as a special case of: 
Lemma 5.1. The following is provable in $\Pi_{1}^{1}-\mathrm{CA}_{0}$. Let $X$ be a separable Banach space. Given a countable set $Y \subset X^{*}$, there is a smallest weak-*-closed set $C \subseteq X^{*}$ such that $Y \subseteq C$.

Proof. We reason in $\Pi_{1}^{1}-\mathrm{CA}_{0}$. For each $x_{0}^{*} \in X^{*}$ and each finite set $F \subset X$, there is a weak- $*$-open set

$$
U\left(x_{0}^{*}, F\right)=\left\{x^{*} \in X^{*} \mid \forall x \in F\left(\left|x^{*}(x)-x_{0}^{*}(x)\right|<1\right)\right\} .
$$

By $\Sigma_{1}^{1}$ comprehension, let $U$ be the weak-*-open set which is the union of the $U\left(x_{0}^{*}, F\right)$ for all $x_{0}^{*} \in X^{*}$ and all finite $F \subset X$ satisfying the arithmetical condition $Y \cap U\left(x_{0}^{*}, F\right)=\varnothing$. In terms of Definitions 4.2 and 4.13 , the code of $U_{n}=U \cap B_{n}\left(X^{*}\right)$ for each $n \in \mathbb{N}$ is the union of the codes of $U\left(x_{0}^{*}, F\right) \cap B_{n}\left(X^{*}\right)$ for all $x_{0}^{*} \in X^{*}$ and all finite $F \subset X$ such that $Y \cap U\left(x_{0}^{*}, F\right)=\varnothing$. Clearly $C=X^{*} \backslash U$ is the smallest weak-*-closed set that includes $Y$. This completes the proof.

For the reversal, we must show that $(\mathrm{S})$ implies $\Pi_{1}^{1}-\mathrm{CA}_{0}$. The standard method of proving that a mathematical statement implies $\Pi_{1}^{1}-\mathrm{CA}_{0}$ is to apply the following lemma $[12,23]$ :

Lemma 5.2. It is provable in $\mathrm{RCA}_{0}$ that the following are equivalent:

1. $\Pi_{1}^{1}-\mathrm{CA}_{0}$;

2. For any sequence of trees $\left\langle T_{n} \mid n \in \mathbb{N}\right\rangle$, there exists a set $W \subseteq \mathbb{N}$ consisting of all $n \in \mathbb{N}$ such that $T_{n}$ is well-founded.

Proof. The assertion that $T_{n}$ is well-founded is $\Pi_{1}^{1}$ (using the sequence $\left\langle T_{n} \mid n \in \mathbb{N}\right\rangle$ as a parameter). The implication from (1) to (2) is therefore obvious. For the converse, reasoning in $\mathrm{RCA}_{0}$, first we show that (2) implies $A C A_{0}$. It is well-known (see [23]) that $\mathrm{ACA}_{0}$ is equivalent over $\mathrm{RCA}_{0}$ to the statement that, for any oneto-one function $f: \mathbb{N} \rightarrow \mathbb{N}$, the range of $f$ exists. Accordingly, let $f: \mathbb{N} \rightarrow \mathbb{N}$ be one-to-one. By recursive comprehension (using $f$ as a parameter), we define a sequence $\left\langle T_{n} \mid n \in \mathbb{N}\right\rangle$ by $s \in T_{n}$ if and only if $\forall k<\operatorname{lh}(s)(n \neq f(k))$. Note that $T_{n}$ is well-founded if and only if $n$ is in the range of $f$. By (2), there is a set $W$ such that $n \in W$ if and only if $T_{n}$ is well-founded, and so $n \in W$ if and only if $n$ is in the range of $f$, i.e., the range of $f$ exists, as desired. This proves $\mathrm{ACA}_{0}$.

Now, reasoning in $\mathrm{ACA}_{0}$, let $\varphi(n)$ be a $\Pi_{1}^{1}$ formula. By the Normal Form Theorem formalized within $\mathrm{ACA}_{0}$ (see [23]), we can write $\varphi(n) \equiv \forall f \exists m \theta(f[m], n)$, where $\theta$ is $\Sigma_{0}^{0}$. Use recursive comprehension to form a sequence of trees

$$
T_{n}=\{s \mid \forall m \leq \operatorname{lh}(s) \neg \theta(s[m], n)\},
$$

$n \in \mathbb{N}$. Note that, for all $n, \varphi(n)$ holds if and only if $T_{n}$ is well-founded. By (2) there exists a set $W$ consisting of all $n$ such that $T_{n}$ is well-founded. Thus for all $n \in \mathbb{N}$ we have $\varphi(n)$ if and only if $n \in W$. This proves $\Pi_{1}^{1}$ comprehension. Thus we have the implication from (2) to (1). This completes the proof.

In showing that the implication from $(S)$ to $\Pi_{1}^{1}-C_{0}$ is provable in $A C A_{0}$, we shall want to know that some of our results from Section 3 are provable in $A C A_{0}$. Our $\mathrm{ACA}_{0}$ version of part of Lemma 3.14 is:

Lemma 5.3. The following is provable in $\mathrm{ACA}_{0}$ (actually $\mathrm{WKL}_{0}$ ). Let $T \subseteq$ Seq be a tree and let

$$
T^{*}=\{s \in \operatorname{Seq} \mid \exists t \in T(t \lll s)\}
$$


be the upward closure of $T$ under majorization. Then $T$ is well-founded if and only if $T^{*}$ is well-founded.

Proof. We reason in $\mathrm{WKL}_{0}$. If $T^{*}$ is well-founded then obviously $T$ is well-founded, since $T \subseteq T^{*}$. Suppose now that $T^{*}$ is not well-founded. Let $f$ be a path through $T^{*}$. By recursive comprehension form the tree $T_{f}=\{t \in T \mid t \ll f[\operatorname{lh}(t)]\}$. Since $f$ is a path through $T^{*}$, we have that for each $n$ there exists $t \in T_{f}$ such that $t \ll f[n]$, and hence $\operatorname{lh}(t)=n$, so $T_{f}$ is infinite. Thus $T_{f}$ is a bounded infinite tree. By Bounded König's Lemma, $T_{f}$ has a path. Here we are using the fact that Bounded König's Lemma is provable in $\mathrm{WKL}_{0}$ (see [12] and Lemma IV.1.4 of [23]). Since $T_{f} \subseteq T$, it follows that $T$ has a path. This completes the proof.

Recall that a tree $T$ is said to be smooth if $T^{*}=T$. The previous lemma implies the following refinement of Lemma 5.2.

Lemma 5.4. It is provable in $\mathrm{ACA}_{0}$ (actually in $\mathrm{WKL}_{0}$ ) that the following are pairwise equivalent:

1. $\Pi_{1}^{1}-\mathrm{CA}_{0}$;

2. For any sequence of trees $\left\langle T_{n} \mid n \in \mathbb{N}\right\rangle$, there exists a set $W \subseteq \mathbb{N}$ consisting of all $n \in \mathbb{N}$ such that $T_{n}$ is well-founded.

3. For any sequence of smooth trees $\left\langle T_{n} \mid n \in \mathbb{N}\right\rangle$, there exists a set $W \subseteq \mathbb{N}$ consisting of all $n \in \mathbb{N}$ such that $T_{n}$ is well-founded.

Proof. The equivalence of (1) and (2) is Lemma 5.2. Given a sequence of trees $\left\langle T_{n} \mid n \in \mathbb{N}\right\rangle$, we can use recursive comprehension to form a sequence of smooth trees $\left\langle T_{n}^{*} \mid n \in \mathbb{N}\right\rangle$. By the previous lemma we have in $\mathrm{WKL}_{0}$ that for all $n, T_{n}$ is well-founded if and only if $T_{n}^{*}$ is well-founded. The equivalence of (2) and (3) follows.

Our $\mathrm{ACA}_{0}$ version of Corollary 3.12 is:

Lemma 5.5. The following is provable in $\mathrm{ACA}_{0}$. Let $S \subseteq$ Seq be such that, for each $s \in S, s^{\frown}\langle m\rangle \in S$ for all but finitely many $m$. Then $Z_{S}$ is a weak-*-closed subspace of $\ell_{1}(\mathrm{Seq})=c_{0}^{*}(\mathrm{Seq})$.

Proof. To simplify notation, let us write $X=c_{0}$ (Seq), so that $X^{*}=\ell_{1}$ (Seq). Clearly $Z_{S}$ is a subspace of $X^{*}$. In order to show that $Z_{S}$ is weak-*-closed, we shall first show that $Z_{S} \cap B_{1}\left(X^{*}\right)$ is weak-*-closed.

By arithmetical comprehension, there exists a sequence $\left\langle M_{s} \mid s \in S\right\rangle$, where $M_{s}$ is the least $M$ such that $s^{\curlyvee}\langle m\rangle \in S$ for all $m \geq M$. Using $\left\langle M_{s} \mid s \in S\right\rangle$ as a parameter, let $\varphi(z)$ be a $\Sigma_{1}^{0}$ formula asserting for $z \in X^{*}$ the existence of $s \in S$ and $M \geq M_{s}$ such that

$$
\left|\frac{1}{s^{\prime}} z(s)-\frac{1}{s} \sum_{m<M} z\left(s^{\frown}\langle m\rangle\right)\right|>\frac{1}{s^{\frown}\langle M\rangle} .
$$

Since $\varphi(z)$ is $\Sigma_{1}^{0}$, it follows by standard $\mathrm{RCA}_{0}$ techniques (see Lemma II.5.7 of [23]) that there exists a relatively weak-*-open set $U \subseteq B_{1}\left(X^{*}\right)$ consisting of all $z \in B_{1}\left(X^{*}\right)$ such that $\varphi(z)$ holds.

We claim that $U=B_{1}\left(X^{*}\right) \backslash Z_{S}$. To see this, let $z \in B_{1}\left(X^{*}\right)$ be given. If $z \notin Z_{S}$, then for some $s \in S$ we have

$$
\left|\frac{1}{s^{\prime}} z(s)-\frac{1}{s} \sum_{m=0}^{\infty} z\left(s^{\frown}\langle m\rangle\right)\right|>0,
$$


whence (1) holds for all sufficiently large $M \geq M_{s}$, whence $z \in U$. Conversely, if $z \in U \cap Z_{S}$, then by (1) we have

$$
\begin{aligned}
& \frac{1}{s^{\wedge}\langle M\rangle}<\left|\frac{1}{s} \sum_{m \geq M} z\left(s^{\wedge}\langle m\rangle\right)\right| \\
& \leq \frac{1}{s} \sum_{m \geq M}\left|z\left(s^{\frown}\langle m\rangle\right)\right| \\
& =\frac{1}{s} \sum_{m \geq M}\left|\frac{s}{s^{\frown}\langle m\rangle} \sum_{n=0}^{\infty} z\left(s^{\frown}\langle m, n\rangle\right)\right| \quad\left(\text { since } z \in Z_{s^{\frown}\langle m\rangle} \text { for all } m \geq M\right. \text { ) } \\
& \leq \frac{1}{s^{\sim}\langle M\rangle} \sum_{m \geq M} \sum_{n=0}^{\infty}\left|z\left(s^{\wedge}\langle m, n\rangle\right)\right| \\
& \leq \frac{\|z\|}{s^{\sim}\langle M\rangle},
\end{aligned}
$$

whence $\|z\|>1$, i.e., $z \notin B_{1}\left(X^{*}\right)$, a contradiction. This proves the claim.

The claim implies that $Z_{S} \cap B_{1}\left(X^{*}\right)$ is weak-*-closed. By Corollary 4.15, it follows in $\mathrm{ACA}_{0}$ that $Z_{S}$ is weak-*-closed. This completes the proof.

We are now ready to prove our main result:

Theorem 5.6 $\left(\mathrm{RCA}_{0}\right)$. The following are pairwise equivalent:

1. $\Pi_{1}^{1}-\mathrm{CA}_{0}$.

2. For every separable Banach space $X$ and countable set $Y \subseteq X^{*}$, there exists a smallest weak-*-closed set in $X^{*}$ containing $Y$.

3. For every separable Banach space $X$ and countable set $Y \subseteq X^{*}$, there exists a smallest weak-*-closed convex set in $X^{*}$ containing $Y$.

4. For every separable Banach space $X$ and countable set $Y \subseteq X^{*}$, there exists a smallest weak-*-closed subspace of $X^{*}$ containing $Y$;

5. For every countable set $Y \subseteq \ell_{1}=c_{0}^{*}$, there exists a smallest weak-*-closed set in $\ell_{1}$ containing $Y$.

6. For every countable set $Y \subseteq \ell_{1}=c_{0}^{*}$, there exists a smallest weak-*-closed convex set in $\ell_{1}$ containing $Y$.

7. For every countable set $Y \subseteq \ell_{1}=c_{0}^{*}$, there exists a smallest weak-*-closed subspace of $\ell_{1}$ containing $Y$.

Proof. The implication from (1) to (2) is just Lemma 5.1. The implications from (2) to (3) and (5) to (6) are straightforward by considering the countable set

$$
\left\{\sum_{i=0}^{n} q_{i} y_{i}^{*} \mid \sum_{i=0}^{n} q_{i}=1, q_{i} \in \mathbb{Q}, q_{i}>0, y_{i}^{*} \in Y, n \in \mathbb{N}\right\} .
$$

The implications from (3) to (4) and (6) to (7) are straightforward by considering the countable set

$$
\left\{\sum_{i=0}^{n} q_{i} y_{i}^{*} \mid q_{i} \in \mathbb{Q}, y_{i}^{*} \in Y, n \in \mathbb{N}\right\} .
$$

The implications from (2) to (5) and (3) to (6) and (4) to (7) are trivial. 
It remains to prove in $\mathrm{RCA}_{0}$ that $(7)$ implies $\Pi_{1}^{1}-\mathrm{CA}_{0}$. First we show in $\mathrm{RCA}$ that (7) implies $\mathrm{ACA}_{0}$. Toward that end, let $f: \mathbb{N} \rightarrow \mathbb{N}$ be one-to-one; we want to show that the range of $f$ exists. Using $f$ as a parameter, let $Y=\left\{y_{\langle n, m\rangle} \mid \exists k \leq\right.$ $m(f(k)=n)\} \subset \ell_{1}$. By (7), let $C$ be the smallest weak-*-closed subspace of $\ell_{1}$ containing $Y$. Claim: for all $n \in \mathbb{N}, y_{\langle n\rangle} \in C$ if and only if $n$ is in the range of $f$. To see this, first suppose $n=f(k)$ for some $k$. Then $y_{\langle n, m\rangle} \in Y$ for all $m \geq k$, and, since $y_{\langle n, m\rangle} \rightarrow y_{\langle n\rangle}$ weak-* as $m \rightarrow \infty$, we have $y_{\langle n\rangle} \in C$. Conversely, suppose $n$ is not in the range of $f$. Then $y_{\langle n, m\rangle} \notin Y$ for all $m \in \mathbb{N}$, and hence, for all $y_{\langle k, m\rangle} \in Y$, $y_{\langle k, m\rangle}(\langle n\rangle)=0$. Let $C^{\prime}$ be the set of all $y \in \ell_{1}$ (Seq) such that $y(\langle n\rangle)=0$. Then $C^{\prime}$ is a weak-*-closed subspace of $\ell_{1}$ which contains $Y$ (and hence contains $C$ ), but $y_{\langle n\rangle} \notin C^{\prime} \supseteq C$. This proves the claim. Now, ' $y_{\langle n\rangle} \in C$ ' is $\Pi_{1}^{0}$ (as $C$ is a code for a weak-*-closed set), whereas ' $n$ is in the range of $f$ ' is $\Sigma_{1}^{0}$, so by recursive comprehension, the range of $f$ exists, as desired. Thus (7) implies $\mathrm{ACA}_{0}$.

So, reasoning within $A C A_{0}$, assume (7). Instead of proving $\Pi_{1}^{1}-C A_{0}$ directly, we shall prove the equivalent statement given by Lemma 5.4 .

Let $\left\langle T_{n} \mid n \in \mathbb{N}\right\rangle$ be a sequence of smooth trees. By recursive comprehension, form the set

$$
T=\left\{\langle n\rangle^{\wedge} s \mid n \in \mathbb{N}, s \in T_{n}\right\} .
$$

Using the notation of Definition 3.6, consider the countable set $Y=\left\{y_{t} \mid t \notin T\right\}$. Note that $Y \subseteq Z_{T}$. By (7) there is a smallest weak-*-closed subspace $C$ of $\ell_{1}$ (Seq) such that $C \supseteq Y$. Since the predicate $y \in C$ is arithmetical (in fact $\Pi_{1}^{0}$, using a code for $C$ as a parameter), we can use arithmetical comprehension to form the set

$$
W=\left\{n \mid y_{\langle n\rangle} \in C\right\} .
$$

We claim that, for all $n \in \mathbb{N}, T_{n}$ is well-founded if and only if $n \in W$.

To prove the claim, let $n$ be such that $T_{n}$ is well-founded. We shall argue by arithmetical transfinite induction on the well-founded tree $T_{n}$ that $y_{\langle n\rangle{ }_{s}} \in C$ for all $s \in$ Seq. (Note that arithmetical transfinite induction is available in $\mathrm{ACA}_{0}$; see Lemma V.2.1 of [23].) The base step consists of observing that $s \notin T_{n}$ implies $\langle n\rangle^{\frown} s \notin T$ which implies $y_{\langle n\rangle-s} \in Y \subseteq C$. For the inductive step, let $s \in T_{n}$ be given such that $y_{\langle n\rangle\urcorner s^{\wedge}\langle m\rangle} \in C$ for all $m \in \mathbb{N}$. Clearly $y_{\langle n\rangle\urcorner s} \wedge\langle m\rangle$ converges weak-* to $y_{\langle n\rangle{ }_{s}}$ as $m \rightarrow \infty$. Since $C$ is weak-*-closed, it follows that $y_{\langle n\rangle{ }_{s}} \in C$. This gives the inductive step. Thus $y_{\langle n\rangle-s} \in C$ for all $s \in$ Seq. In particular $y_{\langle n\rangle} \in C$, i.e., $n \in W$. This proves half of the claim.

For the other half, let $n$ be such that $T_{n}$ is not well-founded. We shall show that $n \notin W$. Let $f$ be a path through $T_{n}$. By recursive comprehension form the set

$$
S=\left\{\langle n\rangle^{\wedge} s \mid f[\operatorname{lh}(s)] \ll s\right\} .
$$

Note that $\langle n\rangle \in S$. Since $T_{n}$ is smooth, we have $S \subseteq T$, and hence $y_{\langle n\rangle} \notin Z_{S} \supseteq Z_{T} \supseteq$ $Y$. Moreover, for any $\langle n\rangle^{\frown} s \in S$, we have $\langle n\rangle^{\frown} s^{\frown}\langle m\rangle \in S$ for all $m \geq f(\operatorname{lh}(s))$, whence by Lemma $5.5 Z_{S}$ is a weak-*-closed subspace of $X^{*}$. It follows that $Z_{S} \supseteq C$, and hence $y_{\langle n\rangle} \notin C$, i.e., $n \notin W$. This completes the proof of the claim.

From our assumption (7) we have shown that for all sequences of smooth trees $\left\langle T_{n} \mid n \in \mathbb{N}\right\rangle$, there exists a set $W$ consisting of all $n$ such that $T_{n}$ is well-founded. Hence by Lemma 5.4 we see that (7) implies $\Pi_{1}^{1}$ comprehension, i.e., (1). This completes the proof of Theorem 5.6.

Theorem $5.7\left(\mathrm{RCA}_{0}\right)$. The following are pairwise equivalent:

1. $\Pi_{1}^{1}-\mathrm{CA}_{0}$. 
2. For every separable Banach space $X$ such that $X^{*}$ is also separable, and for every norm closed subspace $Z \subseteq X^{*}$, there exists a smallest weak-*-closed subspace of $X^{*}$ containing $Z$.

3. For every separable Banach space $X$ such that $X^{*}$ is also separable, and for every weakly closed subspace $Z \subseteq X^{*}$, there exists a smallest weak-*-closed subspace of $X^{*}$ containing $Z$.

4. For every norm closed subspace $Z \subseteq \ell_{1}=c_{0}^{*}$, there exists a smallest weak-*closed subspace of $\ell_{1}$ containing $Z$.

5. For every weakly closed subspace $Z \subseteq \ell_{1}=c_{0}^{*}$, there exists a smallest weak-*closed subspace of $\ell_{1}$ containing $Z$.

Of course, in light of Theorem 3.21, with enough comprehension the equivalences $(2) \Leftrightarrow(3)$ and $(4) \Leftrightarrow(5)$ are trivial; however, we do not know the status of Theorem 3.21 in $\mathrm{RCA}_{0}$.

Proof. Let $X$ be a separable Banach space, with $Z \subseteq X^{*}$ a norm closed subspace. In $\Pi_{1}^{1}-C_{0}$, since $Z$ is norm closed there is a countable set $S \subset X^{*}$ such that $Z$ is the norm closure of $S$ (see [5], [4]). By Theorem 5.6, there is a smallest weak-* closed subspace of $X^{*}$ containing $S$, which must also be the smallest weak-* closed subspace of $X^{*}$ containing $Z$. Thus (1) implies (2). The implications $(2) \Rightarrow(4)$ and $(3) \Rightarrow(5)$ are trivial, and the implications $(2) \Rightarrow(3)$ and $(4) \Rightarrow(5)$ follow from the norm topology being stronger than the weak topology.

It remains only to prove in $\mathrm{RCA}_{0}$ that $(5) \Rightarrow(1)$. As in the proof of Theorem 5.6, we first show that (5) implies $\mathrm{ACA}_{0}$. So assume (5) and let $f: \mathbb{N} \rightarrow \mathbb{N}$ be one-to-one; we want to show in $\mathrm{RCA}_{0}$ that the range of $f$ exists. By recursive comprehension (using $f$ as a parameter), define a tree $T$ by

$$
T=\{\langle\rangle\} \cup\left\{\langle n\rangle^{\urcorner} s \mid \forall k \leq \operatorname{lh}(s)(f(k) \neq n)\right\} .
$$

Then $Z_{T}$ is a weakly closed subspace of $\ell_{1}=c_{0}^{*}$. By (5), let $C$ be the smallest weak-* closed subspace of $\ell_{1}$ containing $Z_{T}$. We claim that for all $n \in \mathbb{N}, n$ is in the range of $f$ if and only if $y_{\langle n\rangle} \in C$. To see this, first suppose $n$ is in the range of $f$, say $f(m)=n$. We'll show by $\Pi_{1}^{0}$-induction (which is available in $\mathrm{RCA}_{0}$; see Corollary 3.10 in [23]) that for all $s \in$ Seq, $y_{\langle n\rangle>s} \in C$. Let $\varphi(i)$ be the $\Pi_{1}^{0}$ formula $\forall s \in \operatorname{Seq}\left(\operatorname{lh}(s)=m-i \rightarrow y_{\langle n\rangle-s} \in C\right)$. By the definition of $T$, if $\operatorname{lh}(s) \geq m$ then $\langle n\rangle \notin T$, whence $y_{\langle n\rangle-s} \in Z_{T} \subseteq C$, and so $\varphi(0)$ holds. Now suppose $\operatorname{lh}(s)=m-i$, and $y_{\langle n\rangle-t} \in C$ for all $t \in$ Seq with $\operatorname{lh}(t)=m-i+1$. Then $y_{\langle n\rangle\urcorner s}\langle k\rangle \in C$ for all $k \in \mathbb{N}$, and $y_{\langle n\rangle\urcorner s<\langle k\rangle} \rightarrow y_{\langle n\rangle-s}$ weak-* as $k \rightarrow \infty$, so $y_{\langle n\rangle\urcorner s} \in C$ since $C$ is weak-* closed. Thus $\varphi(i-1)$ implies $\varphi(i)$, so it follows by $\Pi_{1}^{0}$-induction that $\varphi(i)$ holds for all $i \in \mathbb{N}$. In particular, $\varphi(m)$ holds, i.e., $y_{\langle n\rangle} \in C$. This proves one half of the claim.

Conversely, suppose $n$ is not in the range of $f$; we want to show that $y_{\langle n\rangle} \notin C$. Let $S=\left\{\langle n\rangle^{\frown} s \mid s \in\right.$ Seq $\}$. Then $S \subseteq T$, whence $Z_{S} \supseteq Z_{T}$. It suffices therefore to show that $Z_{S}$ is weak-* closed, because then $Z_{S} \supseteq C$, and $y_{\langle n\rangle} \notin Z_{S}$ since $\langle n\rangle \in S$. In fact, we'll show that $Z_{S}=\left\{z \in \ell_{1}\right.$ (Seq) $\left.\mid \forall t \in S(z(t)=0)\right\}$, which is clearly weak-* closed. Obviously, if $z(t)=0$ for all $t \in S$, it follows from the definition of $Z_{S}$ that $z \in Z_{S}$. On the other hand, suppose $z \in Z_{S}$ and let $t \in S$ be given. Since $z \in Z_{S} \subset Z_{t^{\imath} s}$ for all $s \in$ Seq, it follows that $\left(1 / t^{\prime}\right) z(t)=(1 / t) \sum_{m_{1} \in \mathbb{N}} z\left(t^{\imath}\left\langle m_{1}\right\rangle\right)$, 
which in turn equals $\sum_{m_{1} \in \mathbb{N}}\left(1 /\left(t^{\frown}\left\langle m_{1}\right\rangle\right)\right) \sum_{m_{2} \in \mathbb{N}} z\left(t^{\frown}\left\langle m_{1}, m_{2}\right\rangle\right)$, which equals

$$
\sum_{m_{1}, m_{2} \in \mathbb{N}} \frac{1}{t^{\frown}\left\langle m_{1}, m_{2}\right\rangle} \sum_{m_{3} \in \mathbb{N}} z\left(t^{\frown}\left\langle m_{1}, m_{2}, m_{3}\right\rangle\right),
$$

and so on. Thus for all $k \in \mathbb{N}$ we have

$$
\frac{1}{t^{\prime}} z(t)=\sum_{s \in \mathbb{N}^{k}} \frac{1}{t^{\frown} s} \sum_{m \in \mathbb{N}} z\left(t^{\frown} s^{\frown}\langle m\rangle\right) .
$$

From this it follows easily that

$$
\frac{1}{t^{\prime}}|z(t)| \leq \frac{1}{t^{\frown}\langle 0\rangle^{k}}\|z\|_{1} \quad \text { for all } k \in \mathbb{N},
$$

where $\langle 0\rangle^{k}$ is the sequence of length $k$, all of whose terms are 0 . The right hand side of this inequality approaches 0 as $k \rightarrow \infty$. Thus $z(t)=0$. We have shown that $z \in Z_{S}$ if and only if $z(t)=0$ for all $t \in S$. This completes the proof of the claim. Since ' $n$ is in the range of $f$ ' is $\Sigma_{1}^{0}$ and ' $y_{\langle n\rangle} \in C$ ' is $\Pi_{1}^{0}$, it follows by recursive comprehension that the range of $f$ exists. Thus (5) implies $\mathrm{ACA}_{0}$.

To prove that (5) implies $\Pi_{1}^{1}-\mathrm{CA}_{0}$, we observe that the subspace $Z_{T} \subseteq \ell_{1}$ in the proof of Theorem 5.6 is weakly closed, so we need only repeat the $A_{C A}$ part of that argument. This completes the proof of Theorem 5.7.

\section{REFERENCES}

1. S. Banach, Theory of linear operators, North Holland, 1987, English translation of [2] by F. Jellett. MR 88a:01065

2. Stefan Banach, Théorie des opérations linéaires, Subwencji Funduszu Kultury Narodowej, 1932.

3. Andreas R. Blass, Jeffry L. Hirst, and Stephen G. Simpson, Logical analysis of some theorems of combinatorics and topological dynamics, Logic and Combinatorics (Stephen G. Simpson, ed.), Contemporary Mathematics, no. 65, American Mathematical Society, 1987, pp. 125-156. MR 88d:03113

4. Douglas Kenyon Brown, Functional analysis in weak subsystems of second order arithmetic, Ph.D. thesis, The Pennsylvania State University, 1987.

5. Douglas K. Brown, Notions of closed subsets of a complete separable metric space in weak subsystems of second order arithmetic, Logic and Computation (Wilfried Sieg, ed.), Contemporary Mathematics, no. 106, American Mathematical Society, 1990, pp. 39-50. MR 91i:03108

6. Douglas K. Brown and Stephen G. Simpson, Which set existence axioms are needed to prove the separable Hahn-Banach theorem?, Annals of Pure and Applied Logic 31 (1986), 123-144. MR 87j:03091

7. _ The Baire category theorem in weak subsytems of second order arithmetic, Journal of Symbolic Logic 58 (1993), 557-578. MR 94j:03118

8. Georg Cantor, Contributions to the founding of the theory of transfinite numbers, Dover Publications, Inc., 1955, translated and provided with an introduction and notes by Philip E. B. Jourdain. MR 13:612d

9. Joseph W. Dauben, The trigonometric background to Georg Cantor's theory of sets, Archive for History of Exact Sciences 7 (1971), 181-216.

10. Joseph Warren Dauben, Georg Cantor: His mathematics and philosophy of the infinite, Harvard University Press, 1979. MR 80g:01021

11. Nelson Dunford and Jacob T. Schwartz, Linear operators. Vol. I, Interscience Publishers, Inc., 1958. MR 22:8302

12. Harvey Friedman, Stephen G. Simpson, and Rick Smith, Countable algebra and set existence axioms, Annals of Pure and Applied Logic 25 (1983), 141-181. MR 85i:03157

13. A. S. Kechris and A. Louveau, Descriptive set theory and harmonic analysis, Journal of Symbolic Logic 57 (1992), no. 2, 413-441. MR 93e:04001 
14. Alexander S. Kechris and Alain Louveau, Descriptive set theory and the structure of sets of uniqueness, Cambridge University Press, 1987. MR 90a:42008

15. Alberto Marcone, Foundations of bqo theory and subsystems of second order arithmetic, Ph.D. thesis, The Pennsylvania State University, 1993.

16. Foundations of BQO theory, Transactions of the American Mathematical Society 345 (1994), 641-660. MR 95a:06003

17. S. Mazurkiewicz, Sur la dérivée faible d'un ensemble de fonctionelles linéaires, Studia Mathematica 2 (1930), 68-71.

18. O. Carruth McGehee, A proof of a statement of Banach on the weak-* topology, Michigan Mathematical Journal 15 (1968), 135-140. MR 37:6737

19. Donald Sarason, Weak-star generators of $H^{\infty}$, Pacific Journal of Mathematics 17 (1966), 519-528. MR 35:2151

20. (1968), 129-133. MR 37:3326

21. MR 38:2581

22. Naoki Shioji and Kazuyuki Tanaka, Fixed point theory in weak second-order arithmetic, Annals of Pure and Applied Logic 47 (1990), 167-188. MR 91f:03112

23. Stephen G. Simpson, Subsystems of second order arithmetic, Perspectives in Mathematical Logic, Springer-Verlag, 1996, in preparation.

24. J. von Neumann, Zur Algebra der Funktionaloperationen und Theorie der normalen Operatoren, Mathematische Annalen 102 (1929), 370-427.

Department of Mathematics, The Pennsylvania State University, University Park, Pennsylvannia 16802

E-mail address: jimbo@math.psu.edu

E-mail address: simpson@math.psu.edu 